\title{
EMILIO GABBA E LA BIBLIOTECA DI FAMIGLIA
}

\author{
FRANCESCO BONO (*)
}

SUNTO. - Il saggio esamina genesi e principali caratteristiche della biblioteca della famiglia di Emilio Gabba, mettendo in luce il legame tra la biografia intellettuale e gli interessi dello studioso e i testi presenti nel catalogo. La biblioteca fu creata nel XVIII secolo dai Visconti, notai pavesi profondamente legati all'ambiente culturale cittadino e all'Università. Fra le opere più rilevanti di questo ricco patrimonio librario figurano numerosi testi di storici e pensatori politici, oltre che pubblicazioni di argomento giuridico. Emerge inoltre l'attenzione al pensiero giansenista, diffusosi in concomitanza con le riforme teresiane dell'ateneo.

$$
* * *
$$

ABSTRACT. - The paper discusses the origins and the main features of Emilio Gabba's familial library. It highlights the connection between his interests as a historian and the various books in the catalogue. The library itself was created in the 18th century by the Visconti, a family of notaries from Pavia, deeply connected with the cultural environment of the city and with its university. Among the most important books in this library, many works are written by historians and political thinkers, in addition to several legal texts. A few books underline the family's interesting in Jansenism, which had spread to the university of Pavia, at the same time as the reforms wished by Empress Maria Teresa of Austria.

1. Il titolo racchiude i due poli di questo mio lavoro. Da un lato, Emilio Gabba,${ }^{1}$ con il suo profilo di studioso e la sua umanità, dall'altro una raccolta libraria. Il mio compito è quello di descrivere il legame tra

${ }^{(*)}$ Università degli Studi di Pavia, Italia. E-mail: francesco.bono@unipv.it

1 Per una biografia, anche intellettuale, di Emilio Gabba si vedano i contributi raccolti in: C. Carsana - L. Troiani (a cura di), I percorsi di un historikos. In memoria di Emilio Gabba. Atti del convegno di Pavia (18-20 settembre 2014), Como, New press, 2016; Emilio Gabba: un maestro della storia antica (Roma, 11-12 febbraio 2015), Roma, Bardi, 2017. 
questi due elementi, che chiama in causa le personali memorie dello storico e quelle della sua famiglia.

Emilio Gabba appartiene ad una famiglia dell'élite lombarda del secolo scorso. Il padre Cesare, ingegnere, è fratello di Luigi, ${ }^{2}$ astronomo dell'Osservatorio di Brera, e di Melchiade, militare di carriera, già Ministro dell'Africa italiana. La madre, Maria Franchi Maggi, è la nipote del grande matematico Felice Casorati ${ }^{3}$ e figlia di Emilio Franchi Maggi, ingegnere e sindaco di Pavia per due mandati. ${ }^{4}$

Dal nonno materno proviene una biblioteca di grande interesse storico, appartenuta ai Visconti, notai pavesi del XVIII secolo. ${ }^{5}$ Emilio Franchi, infatti, è nominato erede ${ }^{6}$ da Luigi Felice Maggi, ${ }^{7}$ nipote dei notai Luigi e Gaspare Visconti, originari proprietari, insieme al padre Mauro, della raccolta libraria. La biblioteca Visconti conta più di 1900 titoli ${ }^{8}$ e compete per dimensione con le collezioni librarie cittadine fino

2 E. Gianetto, s.v. Luigi Gabba in DBI 50 (1998), ora disponibile al seguente indirizzo: http://www.treccani.it/enciclopedia/luigi-gabba_\%28Dizionario-Biografico\%29/.

3 E. Togliatti, s.v. Felice Casorati in DBI (1978), ora disponibile al seguente indirizzo: http://www.treccani.it/enciclopedia/felice-casorati_res-3aa57111-87ea-11dc8e9d-016357eee51_(Dizionario-Biografico)/.

4 Sulla famiglia Franchi Maggi: M.C. Brunati, Giuseppe Franchi ed Emilio Franchi Maggi agenti di Pavia, un'illustre dinastia di ingegneri, in Il paese dell'acqua. I Luoghi Pii Elemosinieri di Milano e le loro terre, Milano-Como, Nodo libri, 2013, 312-315.

5 I libri del fondo Visconti sono ora conservati e consultabili presso la Biblioteca Universitaria di Pavia, che ha completato l'opera di inventariazione e di ingressatura nel sistema OPAC (On-line Public Access Catalog) del Sistema Bibliotecario Nazionale (SBN).

6 Sul testamento di Luigi Felice Maggi esiste uno studio di Carlo Francesco Gabba, incaricato di redigere un parere pro veritate da parte di Emilio Franchi Maggi: Intorno al testamento olografo di L.F. Maggi in data 26 aprile 1881. Considerazioni (testamento olografo, datato al principio, composto di parecchie successive disposizioni sottoscritte soltanto), Pisa, Tipografia Nistri, 1887.

7 Per un profilo biografico, A. Gabba, Luigi Felice Maggi pubblico amministratore e conservatore della antiche memorie pavesi, Bollettino della Società Pavese di Storia Patria 112 (2012), 205-210.

8 Il numero di titoli non può in ogni caso essere accostato a quello di biblioteche cardinalizie, per esempio quella di Domenico Passionei, recentemente studiata da: A. Serrai, Domenico Passionei e la sua biblioteca, Milano, Bonnard, 2004. Altri contributi su questa tipologia di raccolta libraria, R. Marzocchi, Biblioteche cardinalizie: $i$ libri del Cardinal Camillo Massimo dallo Studio alla Libraria, in A. Nuovo (a cura di), Biblioteche private in età moderna e contemporanea, Milano, Bonnard, 2005, 117-128; M.C. Misiti, "Pro collegio Patrum Jesuitarum civitatis Viterbii": la libraria del cardinale Scipione Cobelluzzi, in F. Sabba (a cura di), Le biblioteche private come paradigma bibliografico, Roma, Bulzoni, 2008, 195-234. 
ad ora note e studiate, ${ }^{9}$ in una classifica che chiama in causa illustri famiglie patrizie, quali i Bellisomi ${ }^{10} \mathrm{e}$ i Belcredi, ${ }^{11} \mathrm{e}$ personalità di primo piano per l'ambiente cittadino, come Elia Giardini. ${ }^{12}$

É Emilio Gabba stesso, nella Conversazione sulla storia con l'allievo Umberto Laffi, ad inserire la biblioteca tra i ricordi più vividi della sua giovinezza, indugiando in una digressione familiare da lui definita «breve ma curiosa»:

9 A.G. Cavagna, «Il produrre testo proprio stampato è impegnarsi con tutto il mondo»: produzione libraria, editoria e letture nel Settecento pavese, Annali di storia patria, 21 (1992), 309-327, e specc. 323-327 ove l'autrice fornisce alcune notizie su alcune biblioteche private settecentesche. Quanto a cataloghi di raccolte librarie pavesi, A. Nuovo (a cura di), Catalogo del Fondo Antico della Biblioteca del Collegio Ghislieri di Pavia. Edizioni del XV e del XVI secolo, Pavia, Tipografia del Libro, 1983; A. Mauro (a cura di), Catalogo del Fondo Antico della Biblioteca del Collegio Ghislieri di Pavia. Edizioni del XVII secolo, Pavia, Ibis, 1997; E. Grignani, Catalogo delle edizioni pavesi del secolo XVII conservate presso la Biblioteca Bonetta, Bollettino della Società di Storia Patria, 91 (1991), 341-398; M. Forni - A. Gabba, Il lascito Marchesi alla Biblioteca Universitaria di Pavia. Descrizione e valore della raccolta libraria di architettura, Bollettino della società pavese di storia patria, 100 (2000), 263-302; A. Defilippi, La biblioteca di Giuseppe Bertieri, Bollettino della società pavese di storia patria, 117 (2017), 205-296.

10 Sulla biblioteca Bellisomi, A.G. Cavagna, "In ogni mestiere la prima scienza è la cognizione dei libri”. Riflessioni su di una stima libraria del XVIII secolo, in S. Cavaciocchi (a cura di), Produzione e commercio della carta e del libro. Secc. XIII-XVIII, Firenze, Le Monnier, 1992, 449-473; M. Forni, Cultura e residenza aristocratica a Pavia tra '600 e '700, Milano, Franco Angeli, 1989, 157-178.

11 Sulla biblioteca Belcredi, A. Ferraresi, La miscellanea Belcredi, in A. Ferraresi - A. Mosconi Grassano - A. Pasi Testa (a cura di), Cultura e vita universitaria nelle miscellanee Belcredi, Giardini, Ticinensia, Milano, Cisalpino, 1986, 25, 30-31, 42-43; A. Ferraresi, Giuseppe Gaspare Belcredi: un intellettuale di periferia tra riforme e Rivoluzione, in G. De Rosa - F. Agostini (a cura di), Vita religiosa e cultura in Lombardia e nel Veneto nell'età napoleonica, Bari, Laterza, 1990, 276-278.

12 Resta invece esclusa la Biblioteca Universitaria, dato il suo carattere pubblico: M.C. Selva, La Biblioteca Universitaria di Pavia nella seconda metà del Settecento: acquisizioni e cataloghi, Bollettino della Società di Storia Patria, 94 (1994), 195-230. Sulla fondazione di biblioteche pubbliche in Lombardia, S. Furlani, Maria Teresa fondatrice di biblioteche, in A. De Maddalena - E. Rotelli - G. Barbasini (a cura di), Economia, istituzioni, cultura in Lombardia nell'età di Maria Teresa, III, Bologna, Il Mulino, 1982, 1057-1076; M.T. Monti, I libri di Haller e la nascita delle biblioteche pubbliche nella Lombardia asburgica, Società e Storia, 46 (1989), 995-1030; L. Zumkeller, Un mecenate del Settecento e la biblioteca Nazionale Braidense: il cardinale Angelo Maria Durini, e la donazione della sua biblioteca all'istituzione culturale milanese, Il bibliotecario, 26 (1990), 105-114. 
"Nella casa di famiglia, nella periferia di Pavia, dove abitai durante gli anni di guerra..., è conservata una biblioteca appartenuta alla famiglia dalla quale mio nonno materno ereditò parte dei beni... La biblioteca di carattere giuridico, ricca anche di testi storici e letterari greci, latini, italiani e francesi, fornisce un'ottima idea della cultura illuministica a Pavia. Fra l'altro, accanto alle Considerations del Montesquieu vi è la traduzione italiana (Pisa 1779-1786) della Istoria della decadenza e rovina dell'impero romano del Gibbon, che in parte lessi tra i 14 e 15 anni. Per la stesura della mia tesi di laurea mi servii dell'edizione di Polibio del Casaubonus, degli Scriptores rei rusticae veteres latini del Gesner e soprattutto della splendida edizione in folio delle Historiae Romanae Cassii Dionis, curata dal Reimarus". ${ }^{13}$

Le parole di Gabba rivelano già quanto stretta sia la connessione tra la sua biografia e la biblioteca. Inizialmente, infatti, vi trovò le sue prime letture di storiografia, sfogliando la rara traduzione italiana del Gibbon; ${ }^{14}$ poi, come giovane studente universitario impegnato nella stesura della tesi, consultò alcune delle preziose edizioni di storici antichi per scrivere la ricerca sull'esercito romano. ${ }^{15} \mathrm{La}$ biblioteca costituì per lui un utile strumento di lavoro anche successivamente, come dimostra la menzione dell'Historia Romana di Cassio Dione, edita da Reimar, nell'articolo sulla Lex Plotia Agraria, apparso nel $1950 .^{16}$

Gabba sapeva bene che quel patrimonio librario, custodito fra le

13 E. Gabba, Conversazione sulla storia, a cura di U. Laffi, Pisa, 2009, 9.

14 Gibbon, Storia della decadenza e rovina dell'Impero Romano, 8, vol. 9, Pisa, 1779 [E. Gibbon, Istoria della decadenza e rovina dell'impero romano tradotta dall'inglese di Edoardo Gibbon, in Pisa, Ginesi - Grazioli - Raffaelli, 1779-1786]. La storia della traduzione, apparsa anonima, è ripercorsa da: S. Rotta, Il viaggio in Italia di Gibbon, Rivista Storica Italiana LXXIV (1962), 324-354.

15 Ledizione del De lingua Latina di Varrone, curata da Scaliger (M. Terentii Varronis opera quae supersunt. In lib. De ling. Lat. Coniectanea Iosephi Scaligeri, recognita E appendice aucta. In libros De Re rust. notae eiusdem Ios. Scal. non antea editae. His adiuncti fuerunt Adr. Turn. Comment. in lib. De lingua latina: cum emendationibus Ant. Augustini. Item P. Victorii Castigationes in lib. De re rustica, [Ginevra] excudebat Henr. Stephanus, 1573) reca una nota manoscritta di Gabba del 2 dicembre 1944: «I libri del De lingua latina non sono i 4-9 ma i 5-10 per cui bisogna aumentare di un numero la cifra in cima alla pagina».

16 E. Gabba, Lex Plotia Agraria, ora in Id., Esercito e società nella tarda Repubblica romana, Firenze, La Nuova Italia, 1973, 445: «H.S. Reimar, nella sua preziosa edizione commentata di Cassio Dione»; e n. 10: «Cassii Dionis Historiae Romanae quae supersunt, I, Hamburgi 1750, p. 152 \$15». 
mura domestiche, costituiva una testimonianza unica per il contesto pavese, anche in considerazione del fatto che «la storia della cultura nella Pavia del secolo Diciottesimo...è praticamente ancora da fare». ${ }^{17}$ Proprio da tale consapevolezza deriva il suggerimento che mi rivolse, in un pomeriggio di quasi otto anni fa, di studiare il catalogo librario settecentesco della biblioteca. ${ }^{18}$ I risultati di questa ricerca, che allora egli mi propose e che seguì personalmente, sono qui presentati per la prima volta.

L'obiettivo dello studio è far luce sul rapporto tra gli interessi eruditi dei proprietari, i Visconti, membri della "borghesia" del Settecento pavese, e il clima culturale della città, che, a partire dalle riforme universitarie teresiane, conosce un periodo di grande dinamismo e di fioritura. In particolare, si è tentato di capire in che misura gli stimoli e le idee che provengono dall'ambiente cittadino e dall'Università, i cui cortili sono frequentati da personaggi di levatura nazionale, come Tamburini o Gatti, abbiano condizionato le scelte operate dai proprietari, senza trascurare le implicazioni tra quanto accadeva nell'intera penisola, infiammata dal pensiero dei philosophes.

2. Preliminare alla descrizione della raccolta libraria è la ricostruzione delle biografie dei Visconti, condotta sulla base delle risultanze archivistiche.

Mauro Visconti è il capostipite di una numerosa famiglia e può essere considerato il vero fondatore della biblioteca, come attestano le marche

17 Ferraresi, La miscellanea Belcredi, cit., 30.

18 Il documento da cui questa ricerca muove è un catalogo librario settecentesco, il quale fotografa la biblioteca appartenuta alla famiglia Visconti. Esso è ancora oggi conservato presso la famiglia Gabba, e si presenta come un quaderno, in quarto, rilegato in cartone marmorizzato. Esso è composto da 109 fogli, non numerati, scritti fronteretro, da una mano settecentesca, dall'andamento chiaro ed elegante. Il manoscritto è databile verso la fine del XVIII secolo, e più precisamente il termine entro cui fu redatto è il 1799; questa datazione si desume facilmente dal libro più recente in esso contenuto, le Leggi della Repubblica cisalpina (Leggi della repubblica cisalpina dal giorno dell'attivazione della costituzione dell'anno sesto, con i proclami del Direttorio esecutivo, e dei ministri, Milano, dalla Stamperia italiana e francese a S. Zeno n. 534, anno VII repubblicano [1799]). Il catalogo si divide in tre colonne: nella prima sono indicati l'autore, il titolo, il formato, il numero di volumi, luogo e data di edizione; nella seconda lo scaffale, ed infine, nella terza, la segnatura assegnata all'opera. Il catalogo ha come scopo quello di facilitare la consultazione dei testi della biblioteca da parte dei proprietari. 
di possesso manoscritte sul frontespizio di molti libri. Nato intorno al 1730 da Giuseppe Visconti, muore a Pavia il 23 marzo 1796, ${ }^{19}$ quando la città attende l'arrivo delle truppe francesi. Sposa Marianna Comaschi, originaria di Sant'Angelo Lodigiano, dalla quale avrà 8 figli: Luigi, Gaspare, Maria Teresa, Apollonia, Angelo, Angiola, Rosalinda, ${ }^{20}$ Giuseppe. Dopo aver sostenuto l'esame prescritto dai Regolamenti del Collegio Notarile ${ }^{21}$ di Pavia, ${ }^{22}$ Mauro viene iscritto nel Libro delle Matricole del Collegio il 9 settembre del 1758 come publicus notarius civitatis Papiae extra tamen collegium. ${ }^{23}$ L'attività di notaio, alla quale si associa quella di causidico, ${ }^{24}$ verrà svolta «nella città di Pavia e nello studio inferiore corrispondente con tre finestre, un uscio a mattino verso la Corte della casa di abitazione...sita in Porta Palacense, Parrocchia di San'Eusebio» ${ }^{25}$ per quasi quarant'anni, ${ }^{26}$ fino ad un mese prima dell' «immatura morte». ${ }^{27}$

19 Liber defunctorum, Parrocchia di San'Eusebio, ora Parrocchia di San Francesco. È dal registro che reca l'età del defunto, morto a 66 anni, che si ricava il momento della nascita.

20 Rosalinda entra nel convento di Santa Maria delle Cacce di Pavia. Il padre, con atto del 12.2.1791 a rogito del notaio Picotti, provvede alla costituzione della "dote spirituale". Anche la sorella Angiola diventa monaca nello stesso convento l'anno successivo, come si apprende dall'atto di dote del 23.10.1792.

21 Un volume ottocentesco fornisce le prime coordinate normative inerenti all'esercizio della professione di notaio: Cenni dell'origine, de' progressi, della importanza e de' precipui offizii del notariato, Pavia, 1837. Il lavoro di Alberto Liva (Notariato e documento notarile a Milano. Dall'Alto Medioevo alla fine del Settecento, Roma, Consiglio nazionale del notariato, 1979), pur dedicato alla realtà milanese, è comunque valido per la comprensione del notariato pavese. Di recente, S. Levati, Notai e società nello Stato di Milano alla fine d'antico regime (1751-1800), in M. Meriggi - A. Pastore (a cura di), Le regole dei mestieri e delle professioni. Secoli XV-XIX, Milano, Franco Angeli, 2000, 120-151.

22 ARChivio di StATO - PAVIa [d'ora in poi, ASPv], Fondo Notarile Pavia, cart. 16360-II. La domanda di ammissione presentata al Collegio è esaminata nella seduta del 13 luglio 1758. L'esame pubblico si tiene a distanza di qualche mese, il 2 settembre.

23 ASPv, Fondo Notarile, 16360.

24 Era possibile svolgere entrambe le professioni, come chiaramente dimostrato per l'area milanese da: E. Pagano, Avvocati ed esercizio della professione legale in Lombardia nel secondo Settecento. I causidici collegiati di Milano, Rivista di Storia del diritto italiano, 74 (2001), 355-418.

25 ASPv, Fondo Notarile Pavia, 14568. La descrizione dello studio è comunque costante nell'intestazione degli atti rogati da Mauro Visconti a partire dal 1759. Prima di allora Mauro ha cambiato la propria residenza per due volte: prima in Porta Marica e poi in Porta Palatii.

26 ASPv, Fondo Notarile Pavia, 14524 (9.9.1758) - 14568 (15.2.1796).

27 ASPv, Fondo Notarile Pavia, 16360, Supplica Luigi Visconti al Supremo Tribunale di Giustizia. 
È il figlio Luigi, nato intorno al 1760, a farsi carico di continuare lo studio del padre ${ }^{28}$ dopo la sua scomparsa. Iscrittosi all'università di Pavia il 20 novembre del $1775,{ }^{29}$ discute le propositiones per il conseguimento della laurea in utroque iure il 13 maggio del $1790 .^{30}$ Inizia ad esercitare la professione dell'avvocatura, ${ }^{31}$ ma la morte del padre cambia notevolmente la situazione. Il 1796 è infatti un anno di profondi sconvolgimenti nella vita di Luigi, non solo per il lutto famigliare: a seguito dell'insurrezione di Pavia contro le truppe francesi della fine di maggio, egli viene tradotto, come ostaggio, ad Antibes insieme ad altri cittadini pavesi. ${ }^{32}$ La forzata reclusione nella piccola città marittima dura sei mesi, ${ }^{3}$ e eappena fu egli di ritorno alla Patria la prima sua cura fu quella di pensare ai mezzi onde riparare li gravi danni che gli apportarono siffatte disgrazie e di mettersi in istato di poter coll'accostumata decenza sostenere il peso della numerosa famiglia». ${ }^{34}$ Luigi decide quindi di chiedere l'ammissione al Collegio dei Notai di Pavia, ma le condizioni economiche di famiglia costituiscono un imponente ostacolo, dato che il Regolamento Generale per $i$ Notari della Lombardia del 18 marzo 1794 richiede, all'articolo VI, che il candidato al

28 La continuazione dell'attività paterna è provata anche dalla coincidenza della sede dello studio. Luigi, infatti, nelle rituale intestazione dell'atto, ricalca quella utilizzata dal padre: «nella città di Pavia e nello studio inferiore corrispondente con tre finestre ed un uscio a mattina verso la corte della casa di abitazione...posta in Porta Palacense, Parrocchia di Sant'Eusebio in San Francesco Grande, al civico n. 1316» (cfr. ASPv, Fondo Notarile Pavia, 15204).

29 ASPv, Fondo Università, Registro studenti, 810.

30 ASPv, Fondo Università, 855; ASPv, Fondo Università, 616, che contiene la locandina oggi pubblicata in: M.C. Zorzoli, Le tesi legali all'Università di Pavia nell'età delle riforme: 1772-1796, Milano, Istituto Editoriale Cisalpino-La Goliardica, 1980, 154.

31 L'attività di avvocato in Lombardia alla fine del Settecento è stata oggetto di recenti studi: M.G. di Renzo Villata, Un avvocato lombardo tra Ancien Régime e «modernità»: Giovanni Margarita, in A. Padoa Schioppa (a cura di), Avvocati e avvocatura nell'Italia dell'Ottocento, Bologna, Il Mulino, 2009, 425-520. Sulla legislazione recante la disciplina dell'esercizio della professione forense: C. Storti, Avvocati milanesi tra Austria e Italia, in Avvocati e avvocatura, cit., 271-321.

32 Sull'insurrezione di Pavia del maggio 1796 restano fondamentali i lavori di: S. Manfredi, L'insurrezione e il sacco di Pavia nel maggio 1796, Pavia, Frattini, 1900; G. Franchi, Gli ostaggi pavesi ad Antibo, Bollettino della Società di Storia Patria, 7 (1907), 234-235.

33 ASPv, Fondo Notarile Pavia 16360, Supplica Luigi Visconti al Supremo Tribunale di Giustizia.

34 ASPv, Fondo Notarile Pavia 16360, Supplica Luigi Visconti al Supremo Tribunale di Giustizia. 
tabellionato debba dimostrare un reddito annuo di 800 lire milanesi. Per ovviare alla mancanza di questo requisito, egli fa istanza all'Amministrazione Generale della Lombardia perché disponga speciale dispensa, la quale gli viene accordata con decreto del 3 ventoso del $1797 .{ }^{35}$ Il 14 marzo viene quindi cooptato nel Collegio notarile, ${ }^{36}$ ove ricopre anche la carica di Delegato. Muore il 26 giugno 1830, ${ }^{37}$ all'età di settant'anni, avendo nominato erede universale ${ }^{38}$ il nipote Luigi Felice Maggi, figlio della sorella Maria Teresa e dell'Ingegnere Carlo Giuseppe.

Anche Gaspare, di poco più giovane rispetto a Luigi, si dedica agli studi giuridici. Si iscrive così all'Università ${ }^{39}$ ove si addottora il 16 giugno 1790. ${ }^{40}$ Per l'occasione verrà stampato un sonetto, dedicato al suo promotore, Luigi Cremani, ${ }^{41}$ titolare della cattedra di Istituzioni Criminali. Gaspare inizia ad esercitare l'avvocatura e, dopo un primo rifiuto, entra a far parte del Collegio dei Notai ${ }^{42}$ nel maggio del 1802. Svolge tale professione per soli sei anni, ${ }^{43}$ diventando in seguito Conservatore dei Registri Immobiliari. ${ }^{44}$

3. Nella seconda metà del Settecento le riforme teresiane riguardanti l'Ateneo pavese ridisegnano in maniera profonda il ruolo dell'università. ${ }^{45} \mathrm{Il}$ piano di riforme perseguito da Maria Teresa è tal-

35 ASPv, Fondo Notarile Pavia 16360, Supplica Luigi Visconti al Supremo Tribunale di Giustizia, Allegato A.

36 ASPv, Fondo Notarile, 16360.

37 Liber defunctorum, Parrocchia di San Francesco Maggiore.

38 Fondo Famiglia Gabba, Testamento 18.6.1830 Notaio Cristoforo Ferrari.

39 ASPv, Fondo Università. 810, Registro Studenti.

40 ASPv, Fondo Università, 855; ASPv, Fondo Università 620, che contiene la locandina trascritta da: Zorzoli, Le tesi legali all'Università di Pavia nell' età delle riforme, cit., 325.

41 Il componimento è raccolto nella Miscellanea Belcredi: Ferraresi, La miscellanea Belcredi cit., 119 nr. 262.

42 ASPv, Fondo Notarile, 16360.

43 ASPv, Fondo Notarile Pavia, 15270 (5.5.1802) - 15372 (20.11.1808).

44 Questa informazione è desunta da: Cronologico del Ticino, 1810, 63.

45 Sul ruolo svolto dall'Università prima del riformismo asburgico: E. Brambilla, Il «sistema letterario» di Milano: professioni nobili e professioni borghesi dall' età spagnola alle riforme teresiane, in Economia, istituzioni, cultura in Lombardia nell'età di Maria Teresa, cit. III, 79-160; Ead., L'Università di Pavia dalle riforme teresiane all' età francese: alcune linee di interpretazione, in A. Stella - G. Lavezzi (a cura di) Esortazioni alle storie, 
mente incisivo che l'Ateneo pavese vivrà dopo il 1770 «un momento irripetibile nella storia culturale lombarda, italiana ed europea». ${ }^{46}$ Nella Pavia di quegli anni l'Ateneo si trasforma in uno dei centri di cultura cittadini più dinamici, e i docenti universitari ne sono di certo i protagonisti.

Per la famiglia Visconti, il contatto con l'Università è abbastanza ravvicinato, visto che Luigi e Gaspare sono stati studenti presso la Facoltà Legale, una delle quattro previste dal Piano del 1773.47

Partendo dalle opere che i docenti dell'Ateneo danno alle stampe per l'attività didattica, i titoli provenienti dalla Facoltà Legale e presenti nel catalogo sono due: ${ }^{48}$ il De iure criminali, ${ }^{49}$ nell'edizione definitiva

Milano, Cisalpino, 2001, 25-42; G. Guderzo, La riforma dell'Università di Pavia, in Economia, istituzioni, cultura, cit., III, 845-862; M.C. Zorzoli, Università di Pavia (15351796). L'organizzazione dello Studio, in Storia di Pavia. IV. L'età spagnola e austriaca, 1, Pavia, Banca regionale europea, 1995, 427-481. Per la Facoltà di Giurisprudenza, Ead., La Facoltà di giurisprudenza (1535-1796), in Storia di Pavia, IV.1, cit., 483-516; Ead., La formazione dei giuristi lombardi nell'età di Maria Teresa: il ruolo dell'università, in Economia, istituzioni, cultura, cit., III, 743-769; M.G. di Renzo Villata, La formazione del giurista in Italia e l'influenza culturale europea tra sette ed Ottocento. Il caso della Lombardia, in Formare il giurista. Esperienze nell'area lombarda tra Sette e Ottocento, a cura e con un saggio introduttivo di M.G. di Renzo Villata, Milano, Giuffrè, 2004, 4065; Ead., Tra Vienna, Milano e Pavia: un piano per un'Università dall'antico lustro assai decaduta (1753-1773), in A. Romano (a cura di), Gli statuti universitari: tradizione dei testi e valenze politiche. Dall'originarietà degli Studi Generali all'autonomia delle Università degli Studi (secc. XI-XXI), Bologna, CLUEB, 2007, 507-546.

46 E. Gabba, La cultura a Pavia negli anni 1773-1805, in Esortazioni alle storie cit., 3.

47 La riforma dell'Università è attuata attraverso l'adozione di due piani: il Piano di direzione, disciplina ed economia dell'Università di Pavia e il Piano scientifico per l'Università di Pavia. Essi sono pubblicati in Statuti e ordinamenti della Università di Pavia dall'anno 1361 all'anno 1859 raccolti e pubblicati nell'XI centenario dell'Ateneo, Pavia, Tipografia cooperativa, 1925, 201-250. Per un inquadramento della politica universitaria di età teresiana: M.G. Di Renzo Villata, 1740-1765: un declino inarrestabile? Il Senato milanese "recalcitrante" tra misure riformistiche di ripiego e modesti segni di rinnovamento dell'Ateneo pavese, in Almum Studium Papiense. Storia dell'Università di Pavia, 2.1, Milano 2015, 63-82; Ead., 1765-1771: gli anni decisivi per la riforma. Dall'incubazione ai risultati, in Almum Studium Papiense. Storia dell'Università di Pavia, 2.1, cit., 83-114; C. Bussolino, 1771-1780: la riforma attuata, in Almum Studium Papiense. Storia dell'Università di Pavia, 2.1, cit., 115-128.

48 Le opere qui di seguito ricordate sono indicate riportando fedelmente quanto contenuto nel catalogo settecentesco, cui segue, tra parentesi quadre, una breve identificazione del libro, svolta secondo il moderno sistema di citazione.

49 Cremani, De jur. crim., 4, vol. 3, Ticini, 1791 [L. Cremani, De iure criminali libri tres volumen I [-III], Ticini, apud haeredes Petri Galeatii, 1791-1793]. 
pubblicata tra il 1791 e il 1793 di Luigi Cremani, ${ }^{50}$ che fu il promotore di Gaspare nella discussione della tesi di laurea, e la Causa contro il Conte Galli, ${ }^{51}$ allegazione stampata da Alfeno Vario. ${ }^{52}$

Deve essere qui ricordata, accanto al nome del Cremani, anche la copia della Laurea di Maria Pellegrina Amoretti, stampata in occasione del dottorato di quella che fu la prima donna laureata in Italia. ${ }^{53}$ L'esistenza di questo volume nel catalogo attesta la forte curiosità che tale evento, la cui direzione fu delegata al professore toscano, suscitò nell'ambiente cittadino, e non è giudizio infondato paragonarlo ad un «originale e preconfezionato coup de théâtre destinato ad avere un notevole impatto (oggi diremmo) mediatico». ${ }^{54}$

Altro nome del corpo docente pavese, i cui lavori trovano posto sugli scaffali della biblioteca Visconti, è quello di Angelo Teodoro Villa, ${ }^{55}$ allora professore alla Facoltà filosofica. Quest'ultimo fu uno dei pochi ad adeguarsi all'ordine governativo di predisporre testi da adot-

50 Un ritratto del Cremani, professore a Pavia, è disegnato da: E. Dezza, Il magistero di Luigi Cremani e la formazione del giurista nell'età delle riforme, in Formare il giurista cit., 107-172.

51 Vario, Causa contro il Conte Galli, 4 [V. Alfeno, Per Francesco Gervasi napoletano e per li fratelli Amati di Pavia contro il Conte Carlo Galli negoziante piacentino nella R. congregazione di Piacenza a delegazione di S.A.R. il sig. Infante delle Spagne e Duca di Parma etc. etc. etc., s.l.d. (Ticini, 1788)].

52 D. Mantovani, Domenico Alfeno Vario professore di Diritto civile (1780-1789): l'immedesimazione polemica nell'antico. Versione accresciuta sulla base di ulteriori documenti, in Formare il giurista cit., 173-261.

53 Laurea di Pellegrina Amoretti, 4, Pavia [Laurea della signora M. Pellegrina Amoretti cittadina d'Oneglia, in Pavia, presso gli stampatori Porro e Bianchi]. Notizie biografiche su Pellegrina Amoretti sono fornite da: F.P. Casavola, Maria Pellegrina Amoretti, Materiali per una Storia della Cultura Giuridica, 28 (1998), 307-315; G. Visintini, La prima donna giurista in Italia, Materiali per una Storia della Cultura Giuridica, 28 (1998), 317-321.

54 E. Dezza, Il magistero di Luigi Cremani cit., 154. La laurea fu celebrata dal Villa, che pubblicò un volume di poesie: cfr. D. Tongiorgi, Angelo Teodoro Villa e gli insegnamenti letterari nell'Univeristà pavese riformata, Bollettino della Società Pavese di Storia Patria, 96 (1996), 21-34, specc. 21-22.

55 A. Zoncada, Angelo Teodoro Villa, in Memorie e documenti per la storia dell'Università di Pavia e degli nomini illustri che v'insegnarono, Pavia, Bizzoni 18771878, I, 515-517; E. Gabba - D. Magnino, Angelo Teodoro Villa e l'insegnamento della storia nell'Università di Pavia nel Settecento, ora in E. Gabba, Pavia. Domicilium sapientiae, Pavia, New Press, 2000, 57-71; D. Tongiorgi, L'eloquenza in cattedra. La cultura letteraria nell'Università di Pavia dalle riforme teresiane alla Repubblica Italiana. 1769-1805, Bologna, Cisalpino, 1997, 29-48. 
tare per l'insegnamento universitario, e questo spiega la pubblicazione delle Lezioni di eloquenza. ${ }^{56}$ Il docente, tuttavia, ha occasione di comunicare il proprio disappunto per lo scarse vendite di tale testo in una lettera $^{57}$ indirizzata alle autorità austriache, e asserisce che solo tre copie sono state acquistate dagli scolari. Non possiamo dire se quella ricordata nel catologo sia una di queste, nonostante Gaspare risulti essere immatricolato ad un anno dalla pubblicazione. Certo è che si può constatare una predilezione per l'opera di questo docente, del resto comprovata dagli altri suoi libri figuranti nell'elenco. ${ }^{58}$

L'efficacia del magistero universitario, veicolo delle idee di cambiamento che soffiavano in tutta Europa, è ulteriormente testimoniata dai temi discussi in sede di esame finale per il conseguimento del titolo di dottore. Gli studenti si confrontano con i maitre à penser del diritto naturale e con i principali esponenti del pensiero illuministico. Persino Luigi e Gaspare, nei puncta delle loro tesi, discutono della fondatezza delle idee di Grozio, il primo, ${ }^{59}$ e di Hobbes e di Rousseu, il secondo ${ }^{60}$

Nello studio di casa Visconti, così, si possono consultare molti dei libri che i docenti della Facoltà Legale dichiarano di utilizzare per i corsi. Le opere dei maggiori esponenti del pensiero giusnaturalistico sono la base filosofica dei corsi di diritto pubblico, tenuti da De Mays e De Saint Clair, e i Visconti posseggono Les devoir de l'bomme et de

56 Villa, Lezioni d'eloquenza, 8, Pavia [T. Villa, Lezioni d'eloquenza, in Pavia, nella stamperia del R. ed I. Monastero di S. Salvatore, s.d.].

57 La corrispondenza, ora pubblicata in: Tongiorgi, L'eloquenza in cattedra, cit., 38 , non è datata.

58 Idem (Villa),Coluto volgarizzamento, 8, Milano, 1753 [Il rapimento d'Elena del poeta Coluto,... tradotto in versi italiani dall'ab. Angelo-Teodoro Villa... Nuova edizione, accresciuta di varie osservazioni e dell' italiana versione delle orazioni di Gorgia e d'Isocrate intorno ad Elena e dell'Epitalamio per la medesima di Teocrito... Il tutto col testo greco di rincontro, in Milano, nella regioducal corte, 1753]; Idem, Orationes accadem., 8, Ticini [Orationes academicae, Ticini Regii, apud Marcum Antonium Porro, Joseph Bianchi, \& Paschalem Trezzi]; Idem, Oratio funeb. in obitu M. Theresiae, 4, Papiae, 1781 [In obitu Mariae Theresiae augustae Romanorum imperatricis reginae Hungar. et Bohem. ducis Mediolani etc. etc. etc. Oratio funebris ab Angelo Theodoro Villa ... babita jussu praesidum provinciae Ticini in templo divi Thomae IV Id. Januar. a. 1781, Papiae, in typographeo $\mathrm{r}$. et i. Monast. S. Salvatoris].

59 ASPv, 616: bellum secundum Punicum exparte Romanorum non fuisse prorsus iniustum contra Grotium, aliosque contendimus.

$60 \mathrm{ASPv}, 620$ : naturalem hominis flatus contra Hobbesii, ac Rosseau sententiam, socialem esse contendimus. 
citoyens, edizione in lingua francese, del De officio bominis et civis di Pufendorf, ${ }^{61}$ e due edizioni dell'Institutions ecclesiastique del Fleury ${ }^{62}$. Sono poi reperibili i magni auctores, Grozio, ${ }^{63}$ Puferdorf, ${ }^{64}$ Thomasius ${ }^{65}$

61 Puffendorf, De officio Hom. et Civol., Francof. et Lipsiae, 1758 [S. von Pufendorf, De officio hominis, et civis secundum legem naturalem, Francofurti, \& Lipsia, impensis Henr. Lud. Broenneri, 1758].

62 Idem, Droit eccles., 12, vol. 2, Paris, 1711 [C. Fleury, Institution au droit ecclésiastique, à Paris, chez P. Émery, 1711]; Idem, Instit., ou Droit ecclesiast., 8, vol. 2, Paris, 1771 [C. Fleury, Institution au droit ecclesiastique, à Paris, chez Herissant fils, 1771].

63 Grotio, Historia Gotthorum, Vandal., et Longobard., 8, Amstell., 1655 [H. Grotius, Historia Gotthorum, Vandalorum, E Langobardorum, Amstelodami, apud Ludovicum Elzevirium, 1655]; Grotius, De la guerre et de la paix, 8, vol. 3, Haye, 1703 [H. Grotius Le droit de la guerre et de la paix, à La Haye, chez le freres van Dole, 1703]; Idem, De la veritable Relig. Christ., 8, Venetiis, 1768 [H. Grotius, De veritate religionis christianae, Venetia, ex Typographia Novelliana, 1768]; Idem, De imp. sum. potest. circa sacra, 4, vol. 2, Neapoli, 1780 [H. Grotius, De imperio summar. potestatum circa sacra cum scholiis criticis, et chronologicis, Neapoli, typis, ac sumptibus Joseph-Maria Porcelli, 1780]; Idem, Florum sparsis ad jus justin., 8, Neapoli, 1777 [H. Grotius, Hugonis Grotii jurisconsulti, et polybistoris summi florum sparsio ad jus Justinianeum, Neapoli, ex officina Vincentii Manfredii, 1777]; Idem, De jure belli, ac pacis cim notis, 8, vol. 2, Amstellod, 1735 [H. Grotius, De iure belli ac pacis libri tres, Amstelaedami, apud JanssonioWaesbergii, 1735]; Idem, Le droit de la guerre et de la paix avec les notes, 4, vol. 2, Basle, 1768 [H. Grotius, Le droit de la guerre et de la paix par Hugues Grotius, Basle, chez Emanuel Tourneisen, 1768]; Idem, De veritate Relig. Christ. cum notis, vol. 3, 8, Halae Mad., 1739 [H. Grotius De veritate religionis christianae, Halae Magdeburgicae, typiset impensis Orphanotrophei, 1739].

64 Puffendorf, Le Droit de la nature et des gens, 4, Vol. 2, Basle, 1771 [S. von Pufendorf, Le droit de la nature et des gens, ou Système général des principes les plus importants de la morale, de la jurisprudence, et de la politique, à Basle, chez Emanuel Thourneisen, 1771]; Idem, Observat jur univol., 4, Vol. 2, Francof. et Lipsiae, 1744 [S. von Pufendorf, Observationes iuris universi, Francofurti Et Lipsiae Impensis Georgii Conradi Gsellii, 1744]; Idem, De jure nat cum comment., 4, Vol. 4 in 2, Francof., 1759 [S. von Pufendorf, De iure naturae et gentium, libri octo, Francofurti et Lipsiae, ex Officina Knochio-Eslingeriana, 1759].

65 Thomasij, Prudentiae Legislat., 8, Vol. 2, Vratislaviae, 1773 [C. Thomasius, Partem priorem Prudentiae quae comprendit consulatoriam fere ubique ex sensu communi deductam a Christiano Thomasio, Vratislaviae, apud Christ. Fridr. Gutsch., 1773]; Idem, Jurisprudent. divin. cum notis, 4, Halae, 1717 [C. Thomasius, Institutionum jurisprudentiae divinae libri tres, Halae, sumtibus \& typis viduae Christophori Salfeldii, regiminis reg. Boruss. typographi, 1717]; Idem, Jur nat. et gent., 4, Halae, 1718 [C. Thomasius Fundamenta juris naturae et gentium ex sensu communi deducta, Halae \& Lipsiae, typis \& sumtibus viduae Christophori Salfeldii, 1718; Paulo plenior, bistoria juris naturalis, cum duplici appendice, Halae Magdeburgicae, impensis Christophori Salfeldii viduae, 1719]. 
e Burlamaqui, ${ }^{66}$ ma anche i giuristi che trasfusero la riflessione giusnaturalistica in testi destinati agli operatori del diritto, per esempio Heinrich $^{67}$ e Samuel Cocceji ${ }^{68}$ autore del progetto di codificazione prussiana del $1751 .^{69}$

I Visconti sono forniti, inoltre, dei numerosi testi presenti nelle indicazioni che l'amministrazione austriaca fornisce per l'insegnamento, affinchè essi siano adottati nelle lezioni. ${ }^{70}$ Si possono quindi leggere le opere della Giurisprudenza Elegante ${ }^{71}$ (Vinnius, ${ }^{72}$ Voet $^{73}$ Noodt $^{74}$ e

66 Burlamaque, Du droit de la nature et des gens, 8, vol. 8, Yverdon, 1766 [J.J. Burlamaqui, Principes du droit de la nature et des gens, Yverdon, 1766-1768].

67 Idem, Autonomia jur. gent. et pheud., 8, Francof., 1720 [H. von Cocceji, Autonomia juris gentium sive De discrimine civitatis mediatae $\mathcal{E}$ immediatae liberae $\mathcal{E}$ non-liberae, Francofurti ad Viadrum, sumptibus Joh. Godofr. Conradi, 1720]; Idem, Exercitat. Curios., 4, vol. 4, Lemgoviae, 1722 [H. von Cocceji, Exercitationum curiosarum ... volumen primum [secundum], Lemgoviae, typis et impensis Henrici Wilhelmi Meyeri, 1722].

68 Coccej, Jus civile controvers., 4, vol. 2, Lipsiae, 1766 [S. von Cocceji, Jus civile controversum, Lipsiae, sumtibus haered. M.G. Weidmanni et Reichii, 1766].

69 Code Frederic le Roi de Pruss., 8, vol. 3,1751 [Code Frederic; ou Corps de droit, pour les etats de sa majeste le roi de Prusse, 1751-1752].

70 I titoli sono ricordati in: M.G. Di Renzo Villata, La formazione del giurista in Italia e l'influenza culturale europea tra sette ed Ottocento. Il caso della Lombardia, in Formare il giurista, cit., 54-60; Dezza, Il magistero di Luigi Cremani, cit., 121-125.

71 Sulla Scuola Elegante, I. Birocchi, Alla ricerca dell'ordine. Fonti e cultura giuridica nell' età moderna, Torino, G. Giappichelli, 2002, 58-69.

72 Vinnij, Comment. Institut., 4, Vol. 2, Venet., 1761 [A. Vinnen, In Quatuor Libros Institutionum imperialium commentarius Academicus, \& Forensis, Venetiis, ex Typographia Balleoniana, 1761]; Idem, Quaest., 12, Marburgi, 1755 [A. Vinnen, Quaestiones iuris selectae, usui forensi potissimum adcommodatae, Marburgi, Sumptibus Christ. Ernst. Immanuel Weldige, 1755]; Idem, Tract et quaest. selectae, 4, ad Rhenum, 1722 [A. Vinnen, Tractatus quinque, de pactis, jurisdictione, collationibus, transactionibus, et quaestionibus juris selectis, Trajecti ad Rhenum, apud Guilielmum vande Water, academiae typographum, 1722].

73 Voet, De famil herusc., 8, Basilae, 1760 [J. Voet, De Familia Eriscunda, liber singularis, Basileae, impensis Thurnisii, J. Rud. Filii, bibl. \& Typ, 1760]; Idem, Pandect., 8, Vol. 2 in 1, Patavij, 1776 [J. Voet, Compendium Juris juxta seriem pandectarum, Patavii, typis Seminarii apud Joannem Manfrè, 1776 ]; Idem, De jure milit., 12, Ultrajecti, 1658 [J. Voet, De Jure militari liber singularis, Ultrajecti, Ex officina Antonii Smytegelt, bibliop., 1670]; Idem, Ad Pandect., 4, Vol. 7, Napoli, 1778 [J. Voet, Commentarius ad Pandectas, Neapoli, Ex Typographia Ursiniana, 1778].

74 Nood, Opera omnia, 4, Vol. 4, Napoli, 1786 [G. Noodt, Opera omnia, Neapoli, apud fratres Terres, 1786]. 
Van Bynkershoek ${ }^{75}$ ) come pure quelli dell'Usus modernus Pandectarum, ${ }^{76}$ (Struve, ${ }^{77}$ Stryk ${ }^{78}$ Böhmer $^{79}$ e Leyser ${ }^{80}$ ), nei cui lavori «le soluzioni venivan sempre meno fondate sull'autorità dei testi romani $\mathrm{o}$ della dottrina del diritto comune, mentre al contrario si preferiva giustificare le teorie più tradizionali col ricorso alla naturalis ratio». ${ }^{81} \mathrm{Da}$ ricordare per completezza l'elaborazione sistematica di Domat ${ }^{82}$ le

75 Bynkershock, Observol. jur. rom., [C. van Bynkershoek, Observationum iuris Romani libri quatuor, Francofurt et Lipsiae, ex officina Krugiana, 1739].

76 F. Wieacker, Storia del diritto privato moderno, I, Milano, Giuffrè, 1980, $305-376$.

77 Idem, Iurisprud., 8, Francof., 1733 [G.A. Struve, Jurisprudentia RomanoGermanica Forensis, Francofurti Ad Moenum, apud Franciscum Varrentrapp, 1733]; Idem, Syntagma jurisprud., 4,Coloniae, 1709 [G.A. Struve, Syntagma jurisprudentiae, secundum ordinem pandectarum concinnatum, Coloniae Agrippinae, apud Franciscum Metternich, 1709]; Idem, Iurisprudentia cum notis, 4, Francof., 1760 [G.A. Struve, Jurisprudentia romano-germanico-forensis, Francofurti ad Moenum, apud Franciscum Varrentrapp, 1760]. Sul giurista, J. Finzel, Georg Adam Struve (1619-1692) als Zivilrechter, Frankfurt am Main, Lang, 2003. Del figlio, Burkhard Gotthelf Struve, bibliotecario e storico, sono conservati due edizioni della sua Introductio in notitia rei litterariae (1678; 1679), la Iuris publici prudentia (1679) e l'Antiquitatum Romanarum syntagma (1677).

78 Strykij, Opera omnia jurid., fol., Vol. 14, Francof., 1743 [S. Stryk, Opera omnia tam tractatus quam disputationes hactenus etiam nondum coniunctim editas, Francofurti \& Lipsiae, sumptibus Samuelis Wohleri, typis Eliae Danielis Sussii, 1743-1753].

79 Boehmeri, Introduct. In jus digest., 8, vol. 2, Halae Magdeb., 1756 [J.H. Böhmer, Introductio in ius digestorum sensum pariter ac vsum bodiernum singularum doctrinarum succincte exbibens, Halae Magdeburgicae, impensis Orphanotrophei, 1756]; Idem, Jus pub. univol., 8, cum notis, Francof., 1758 [ J.H. Böhmer, Introductio in ius publicum universale ex genuinis iuris naturae principiis deductum, Francofurti et Lipsiae, in officinis Trattnerianis, 1758]; Idem, Jus ecclesiasticum, 4, vol. 8, Halae, 1763 [J.H. Böhmer, Ius ecclesiasticum protestantium usum bodiernum iuris canonici, Halae Magdeburgicae, impensis Orphanotrophei, 1756-1763]; Idem, Jus parochiale, 4, Halae, 1760 [J.H. Böhmer, Ius parochiale ad fundamenta genuina revocatum, Halae Magdeburgicae, mpensis Orphanotrophei, 1760]; Idem, Doctrina, de act. cum notis, 8, Francof., 1756 [J.H. Böhmer, Doctrina de actionibus, Francofurti ad Moenum, ex officina buchneriana, 1756].

80 Leyseri, Meditat. ad pandect., 4, vol, Lipsiae, 1772 [A. von Leyser, Meditationes ad Pandectas, Lipsiae, Brunsvigae et Guelpherbyti, apud Meisnerianos fratres, 1772-1786].

81 Wieacker, Storia del diritto privato moderno, cit., 330.

82 Domat, Les loix civiles, fol. vol. 2, Paris, 1766 [J. Domat, Les Loix civiles dans leur ordre naturel; le Droit public, et Legum delectus, à Paris, chez la veuve Cavelier, rue S. Jacques, au Lis d'or, 1766]. 
Pandectae Justinianae di Pothier ${ }^{83}$ e le validissime sintesi di Heinecke. ${ }^{84}$ La biblioteca reca impressi gli echi di un importante movimento culturale sviluppatosi tra i portici dell'Università, il Giansenismo. Sotto la lettera A, quinta riga, in un'elegante calligrafia, viene trascritto un titolo fortemente evocativo di una fondamentale stagione della cultura pavese: si tratta degli Acta et decreta synodi dioecesanae Pistoriensis, ${ }^{85} \mathrm{la}$ traduzione latina pubblicata a Pavia degli Atti del Sinodo di Pistoia del 1786, condannati con la bolla papale Auctorem fidei del 1794, anch'essa registrata dalla stessa mano qualche foglio più avanti. ${ }^{86}$ In queste due opere è racchiuso lo zenit dell'esperienza giansenistica in Italia e, al contempo, l'inizio della sua fine, attraverso un percorso strettamente legato alle vicende dell'università di Pavia.

Negli Atti del Sinodo si cela infatti il lavoro di teologo di Tamburini ${ }^{87}$ allora professore di morale e di teologia fondamentale

83 Pandectae Iustin cum notis, fol., Vol. 3, Parisiis, 1748 [R.J. Pothier, Pandectae Justinianeae, in novum ordinem digestae, Parisiis, apud Saugrain patrem, J. Desaint \& C. Saillant; Carnuti, F. Le Tellier, 1748-1752].

84 Heinneccij, Opera omnia, 4, vol. 9, Geneve, 1771 [J.G. Heinecke, Opera omnia nunc denuo edita, Genevae, sumptibus fratrum de Tournes, 1766-1771]; Idem, Responsa jur., 4, Vratislaviae, 1744 [J.G. Heinecke, Responsa iuris super feudis Langharum pot. Sardin. regi cessis, Vratislaviae, impensis Io. Iacobi Kornii, 1744].

85 Acta et decreta Synodi Pistor., 8, vol. 2, Papia, 1789 [Acta et decreta synodi dioecesanae Pistoriensis an. 1786. Pars I [II], Ticini, sumptibus Balthassaris Comini, 1789]. Per un'analisi delle fonti del Sinodo, si rinvia a: P. Stella (a cura di), Atti e decreti del concilio diocesano di Pistoia dell'anno 1786, II. Introduzione storica e documenti inediti, Firenze, L. S. Olschki, 1986.

86 Sanctissimi D. N. Domini Pij divina Providentia, Romae et Parmae, 1794 [Sanctissimi domini nostri domini Pii divina providentia papae Sexti damnatio quamplurimum propositionum Exceptum ex libro Italico idiomate impresso sub titulo: Atti, e decreti del Concilio diocesano di Pistoja dell'Anno 1786, in Pistoja per Atto Bracali stampatore vescovile, Romae, ex typographia Revol. Camerae Apostolicae, 1794]. Sulla condanna del Sinodo di Pistoia da parte di Pio VI, P. Stella, Il giansenismo in Italia. 2. Il movimento giansenista e la produzione libraria, Roma, Edizioni di Storia e letteratura, 2006, 433-502.

87 Su Tamburini, G. Mantese, Pietro Tamburini e il giansenismo bresciano, Brescia, Ancora, 1942; P. Corsini - D. Montanari (a cura di), Pietro Tamburini e il giansenismo lombardo. Atti del Convegno internazionale in occasione del $250^{\circ}$ della nascita, Brescia, 25-26 maggio 1989, Brescia, Morcelliana, 1993. Per la corrispondenza epistolare tra Tamburini e De' Ricci: P. Guerrini, Carteggi bresciani inediti sula vita e sui tempi di Pietro Tamburini (1737-1827), Bollettino delle Società pavese di storia patria, 27 (1927), 161-250; R. Mazzetti, Relazioni fra il giansenismo pavese e il giansenismo toscano, in "Miscellanea pavese", Torino, Società storica subalpina, 1932, 188-207. 
nella riformata Facoltà Teologica di Pavia ${ }^{88}$ considerato il massimo esponente del giansenismo lombardo. Del resto, gli intensi rapporti tra il teologo bresciano e Scipione De' Ricci, Vescovo di Pistoia sono riccamente documentati. ${ }^{89}$ Proprio in una letterea indirizzata al docente pavese, il presule toscano riconosce allo stesso un ruolo di primo piano nella preparazione del Sinodo, tanto da scrivere: «Ormai Pistoia si valuta un'appendice di Pavia».90

La radicale riorganizzazione dell'Ateneo tocca il portico teologico, ove il legislatore teresiano ritiene di poter trovare una sponda "intellettuale" alla propria politica giurisdizionalista. Tamburini, ${ }^{91}$ insieme a

88 M. Bernuzzi, La facoltà teologica dell'Università di Pavia nel periodo delle riforme (1767-1797), Milano, Cisalpino, 1992. Testimonianza diretta è quella contenuta in Giuseppe Frank Memorie, a cura di G. Galli; presentazione di P. Mazzarello, I, Milano, Cisalpino, 2006, 161: «La facoltà di Teologia era ultra-giansenista, a Roma era considerata eretica e contava tre scienziati di prim'ordine: Natali, Tamburini e Zola. Gli ultimi due dirigevano il Collegio tedesco, nel quale erano accettati soltanto gentiluomini tedeschi che intendevano dedicarsi alla teologia, In precedenza il collegio era a Roma, ma l'imperatore Giuseppe lo fece trasferire a Pavia perché voleva avere dei sacerdoti educati secondo i suoi principi. Gli allievi vivevano nel lusso e si comportavano in maniera esemplare. Per ordine del sovrano, fu aperto anche un Seminario per gli Italiani. I seminaristi erano trecento, ricevevano un' istruzione generale ed erano ben più illuminati dei giovani sacerdoti che uscivano dai seminari diocesani».

89 Sempre di Scipione de' Ricci: Idem pastorale del Vescovo di Pistoia, 8, Pavia, 1788 [S. de' Ricci, Lettera pastorale di Monsignor Vescovo di Pistoja e Prato al clero e al popolo della citta e diocesi di Prato, Edizione seconda, Pavia, Giuseppe Bolzani, 1788].

90 Lettera Scipione de' Ricci a Tamburini 29.5.1786, citata in: P. Vismara, Echi e riflessi del Sinodo di Pistoia in Lombardia, ora in Ead., Settecento religioso in Lombardia, Milano, NED, 1994, 217.

91 Numerosi i testi del teologo pavese segnati nel catalogo Visconti: Idem di un teologo piacentino, 8, vol. 3, Piacenza, 1782 [P. Tamburini, Lettere di un teologo Piacentino a Monsignor Nani vescovo di Brescia sul rumore eccitato da alcuni suoi Teologi contra l'Analisi de libro delle Prescrizioni di Tertulliano, in Piacenza e si vende in Cremona presso Lorenzo Manini, ed altri libraj, 1782-1785]; Tamburini, Apologia del libro De Tollerantia, 8, Piacenza, 1786 [P. Tamburini, Riflessioni del Teologo Piacentino sul libro dell'ab. Cuccagni De mutuis Ecclesiae E Imperii officiis Ec. stampato in Roma contra l'opera del sig. conte di Trautmansdorf De tolerantia ecclesiastica E civili, nelle quali si prende occasione di rischiarare alcuni principj intorno al diritto de' principi sulle cose ecclesiastiche, in Piacenza, e si vende in Cremona, presso Lorenzo Manini ed altri libraj, 1786]; Idem, Origene, 8, Pavia, 1786 [P. Tamburini, Ragionamenti sul primo libro di Origene contro Celso..., Pavia, P. Galeazzi, 1786]; Idem, Ethica Christiana, 8, Vol. 4, Ticini, 1783 [P. Tamburini, Petri Tamburini brixiani Praelectiones de iustitia christiana et de sacramentis, Ticini, in typographeo Petrii Galeatii, 1783-1788]. 
Natali e Zola, ${ }^{92}$ interlocutori di primo piano nel dibattito sul giansenismo, vengono così ad insegnare nella Facoltà teologica, e «la fiducia concessa dallo Stato a questi teologi non fu mal riposta». ${ }^{93}$ I professori giansenisti si schierano a favore dei programmi di Maria Teresa pubblicando opuscoli dai quali esplicitamente si deduceva la loro adesione al giurisdizionalismo.

Nella biblioteca Visconti si potevano così leggere il De tolerantia di Tamburini, ispirato dall'emanazione della Patente di tolleranza del $1781,{ }^{94}$ accanto ai principali lavori del regalismo, quali l'Istoria civile del regno di Napoli del Giannone ${ }^{95}$ e Di una riforma d'Italia del Pilati, ${ }^{96}$ «l'altro e maggior libro emerso nella pubblicistica giurisdizionale degli anni settanta». ${ }^{97}$ In questo senso non stupisce rinvenire le opere del Sarpi, ${ }^{98}$ la cui Storia del Concilio è in cinque copie.

92 A. Zambarbieri, Le goût de l'histoire. Giuseppe Zola agli esordi del suo insegnamento pavese, in S. Negruzzo (a cura di), Il giansenismo e l'Università di Pavia. Studi in ricordo di Pietro Stella, Milano, Cisalpino, 2012, 115-132.

93 Bernuzzi, La facoltà teologica, cit. 134.

94 Idem, De Tollerantia, 8, Ticini, 1783 [P. Tamburini, Thaddaei S.R.I. comitis de Trautmansdorf ... De tolerantia ecclesiastica et civili. Ad Iosephum II Augustum, Ticini, in typographeo Petri Galeatii, 1783].

95 Giannone, Storia del Regno di Napoli, 8, vol. 4, Ven., 1766 [P. Giannone, Istoria civile del regno di Napoli, in Venezia, presso Giambatista Pasquali, 1766]; Idem, Storia di Napoli, 8, vol. 16 in 8, Nap., 1770 [P. Giannone, Istoria civile del Regno di Napoli, Napoli, nella stamperia di Giovanni Gravier, 1770]. Presenti anche due edizioni delle Opere postume: Idem, Opere postume, 8, vol. 2, Napoli, 1773 [P. Giannone, Opere postume di Pietro Giannone colla di lui vita, Napoli, nella stamperia di Giovanni Gravier, 1773]; Idem, Opere postume, 8, Venezia, 1768 [P. Giannone, Opere postume di Pietro Giannone in difesa della sua Storia civile del regno di Napoli con la sua professione difede, in Venezia, presso Giambatista Pasquali, 1768]. Per una biografia intellettuale di Giannone rimane fondamentale: G. Ricuperati, L'esperienza civile e religiosa di Pietro Giannone, Milano, Ricciardi, 1970; Id., La città terrena di Pietro Giannone. Un itinerario tra 'crisi della coscienza europea' e illuminismo radicale, Firenze, L.S. Olschki, 2001.

96 Riforma d'Italia, 8, Vol. 3, Londra, 1786 [C.A. Pilati, Nuovo progetto d'una riforma d'Italia, ossia Dei mezzi di liberar l'Italia dalla tirannia de' pregiudizi e della superstizione; col riformarne i piu cattivi costumi e le più perniciose leggi, Londra, appresso C. Thompson, 1786]. Dello stesso autore anche: Pilati, Esistenza della legge natur., 8, Venezia, 1764 [C.A. Pilati, L'esistenza della legge naturale impugnata, e sostenuta da Carlantonio Pilati, in Venezia, presso A. Zatta, 1764].

97 F. Venturi, Settecento riformatore, II, Torino, Einaudi 1990, 250.

98 Sarpi, Opere, 12, Vol. 6, Venezia, 1685 [P. Sarpi, Opere, in Venetia, appresso Roberto Meietti, 1677]; Idem, Storia del Concilio Trident. colle note, 4, Vol. 2, Londra, 
Gli scritti dei professori hanno a cuore problematiche teologiche e di natura più prettamente ecclesiale. Nei loro lavori essi spalancano le porte a molti degli autori di area porto realista e giansenista: per la censura ecclesiastica sono solo libri all'Indice, per i docenti pavesi sono le fonti delle lezioni pubblicate. Da parte sua, anche il governo mostra una propensione verso le opere di matrice giansenistica, e consiglia la loro lettura agli studenti del Collegio Germanico-Ungarico, ${ }^{99}$ di cui, guarda caso, Tamburini è prefetto agli studi. Entro questa cornice va quindi a collocarsi il ritrovamento sugli scaffali dei Visconti di molti pensatori di Port Royal, senza dimenticare che in tutta la penisola la circolazione dell'editoria giansenista è fenomeno assai diffuso e capillare. Il mercato librario offre sia i «testi confluiti nella penisola e nelle isole maggiori» sia la «produzione propriamente italiana, distinta a sua volta in traduzioni e dal francese e dal latino e in scritti originali, dovuti soprattutto alla penna di esponenti del movimento giansenista italiano». ${ }^{100}$ Oltre alle immancabili Provinciali di Pascal, ${ }^{101}$ tra i preferiti dai nostri notai pavesi spicca D'Arnaud, ${ }^{102}$ il teologo francese sostenitore di Giansenio, con la sua opera più famosa, De la fré-

1757 [P. Sarpi, Istoria del concilio tridentino, in Londra, alle spese dei fratelli De Tournes, 1757]; Idem, Opere, 4, Vol. 8, Verona, 1768 [P. Sarpi, Istoria del Concilio Tridentino, in Helmstat, per Jacopo Mulleri, 1761-1768]; Idem, Opere, 8, Vol. 24 in 12, Napoli, 1789 [P. Sarpi, Raccolta delle opere, Napoli, 1789-1790; Istoria del Concilio Napoli, sii vendono presso Giuseppe Onorato, 1789-1790]; Idem, Historiae Concilij Trident., 4, Vol. 3 , Lipsiae, 1690 [P. Sarpi, Historia Concilii Tridentini, libri octo exposita, Lipsiae, impensis Martini Theodori Heybey, 1690].

99 Le regole del Collegio Germanico-Ungarico sono incluse nella Collezione di I.R. leggi ed ordinanze in materie economiche ecclesiastiche dall'anno MDCCLXVII fino al MDCCLXXXIII. Tradotte dal tedesco, in Pavia, nella Stamperia del Regio, ed Imperial Monistero di S. Salvatore, 1785, attestata nel catalogo di casa Visconti. Dall'estratto pubblicato da Stella (Il giansenismo in Italia, II, cit., 383-384) si possono individuare facilmente gli autori di matrice portorealista suggeriti: Nicole, Bossuet, Fleury, Arnaud, Sacy. È questo un ulteriore indizio del clima culturale che la Pavia teresiana e giurisdizionalista viveva.

100 Stella, Il giansenismo in Italia, II, cit., 61.

101 Montalte, Lettres provinc., 8, vol. 3, Amsterd., 1735 [B. Pascal, Les provinciales, 1733].

102 Dello stesso autore anche: Idem, Lettres, 12, vol. 9, Nancy, 1743 [A. Arnauld, Lettres, à Nancy, aux depens de Joseph Nicolai, 1727-1743]; Arnaldo, Della proibizione dei libri, 8, 1771 [A. Arnauld, Delle proibizioni de' libri, in Venezia, 1771]; Perpetuité de la Foy, 12, Paris, 1672 [A. Arnauld, La perpetuité de la foy de l'Eglise Catholique touchant l'eucharistie, à Paris, chez la veuve Charles Savreux, libraire jure, au pied de la tour de N. Dame, a l'enseigne des trois vertus, 1672]. 
quente Communion. ${ }^{103}$ Potevano essere inoltre consultate le opere di Helvetius ${ }^{104}$ e D'Holbach, ${ }^{105}$ Febronius, ${ }^{106}$ Eybel,${ }^{107}$ Nicole, ${ }^{108}$ Fleury ${ }^{109}$ e

103 Idem, De la frequente communion, 8, Paris, 1648 [A. Arnauld, De la fréquente Communion, à Paris, chez Antoine Vitré, 1648].

104 De l'Esprit, 8, vol. 2, Amsterd., 1776 [C.A. Helvetius, De l'esprit, à Amsterdam \& à Leipsick, chez Arkstee et Merkus, 1776]; Helvetius, Le Borcheur Poeme, 8, Londres, 1573 [C.A. Helvetius, Le bonheur, poeme en six chants... Ouvrages posthumes, à Londres, 1773]. Il pensiero di Hélvetius era stato ripreso a Milano, tra gli anni '60 e '70 del Settecento, da Pietro Verri e da Giuseppe Gorani: F. Venturi, Settecento riformatore, V, L'Italia dei lumi, 1, Torino, Einaudi, 1987, 501; G. Francioni, Prefazione, in P. Verri, Meditazioni sulla felicità, Como-Pavia, Ibis, 2013, 9-50.

105 Christianisme devoilée ou examen, 12, Londres, 1761 [P.H.D. d'Holbach, Le Christianisme dévoilé ou Examen des principes et des effets de la religion chretienne, à Londres, 1767]; De l'Imposture sacerdotale, 12, Londres, 1767 [P.H.D. d'Holbach, De l'imposture sacerdotale ou Recueil de pieces sur le clerge, Londres, 1767]; Mirabaud, Systeme de la nature, 8, vol. 2 in 1, Lond., 1781 [P.H.D. d'Holbach, Systeme de la nature, ou des loix du monde physique et du monde moral, à Londres, 1781]; Morale universelle, 8, vol. 3, Amsterd., 1776 [P.H.D. d'Holbach, La morale universelle. Ou les devoirs de l'homme fondes sur sa nature, à Amsterdam, chez Marc-Michel Rey, 1776]; Systeme Social, 8, Vol. 3 in 1, Londres, 1773 [P.H.D. d'Holbach, Systeme social. Ou principes naturels de la morale et de la politique, Londres, 1773].

106 Febronij, Jur pub ecclesiastici, 8, Hulmae, 1767 [J.N. von Hontheim, Principia juris publici ecclesiastici catbolicorum ad statum Germaniae, Ulmae, T. Bartholomaei, 1767]; Idem, Govern. de l'Eglise, 8, vol. 3, Venise, 1766 [J.N. von Hontheim, Traite du gouvernement de l'eglise, et de la puissance du pape, par rapport a ce gouvernement, chez Pierre Remundi, 1766-1767]; Idem, De Statu Ecclesiae, 4, Bullioni, 1765 [J.N. von Hontheim, De statu Ecclesiae et legitima potestate Romani pontificis liber singularis, Bullioni, apud Guillielmum Evrardi, 1765]. s.1., 1782].

107 Cosa è il Papa tradotto dal tedesco, 12, 1782 [G.V. Eybel, Cos'è il papa?,

108 Nicole, Perpetuità della Fede, 12, Venezia, 1779 [P. Nicole, La perpetuità della fede della chiesa cattolica rapporto all'eucaristia, in Venezia, presso Lorenzo Baseggio, 1779].

109 Fleury, Discours sur les libertez Gallican., 8, Hayae, 1755 [C. Fleury, Maximes et libertez gallicanes, rassemblées et mises en ordre, avec leurs preuves, à La Haye, 1755]; Idem, Discours sur l'Histoire eccl., 12, Paris, 1742 [C. Fleury, Discours sur l'bistoire ecclesiastique, à Paris, chez Montalant, 1742]; Idem, Sopra la Storia ecclesiastica, vol. 2 in 1, 12, Ven., 1772 [C. Fleury, Discorsi sopra la storia ecclesiastica, in Venezia, presso Antonio Zatta, 1772]; Idem, Histoire eccl., 12, vol. 36, Paris, 1742 [C. Fleury, Histoire ecclesiastique, à Paris, chez Montalant, 1742; Histoire ecclesiastique pour servir de continuation à celle de feu par mr. l'abbe Fleury, à Paris, chez Montalant, 1742]; Idem, Droit eccles., 12, vol. 2, Paris, 1711 [C. Fleury, Institution au droit ecclésiastique, à Paris, chez P. Émery, 1711]; Idem, Les Moeures des Israelites, 8, vol. 2 in 1, Brux., 1777 [C. Fleury, Les moeurs des israelites, à Bruxelles, et se vend à Liege, chez Jean-Francois 
Boussuet $^{110}$. Per la storia ecclesiastica si può ricorrere a Racine ${ }^{111} \mathrm{e}$ Berruyer, ${ }^{112}$ poi all'Histoire du vieux et de noveau testament di Nicolas Fontaine. ${ }^{113}$ Non casuale il rinvenimento di opere del canonista Riegger, considerato uno dei «teorici delle chiese nazionali». ${ }^{114}$

La biblioteca conserva poi parecchie opere di pensatori settecenteschi, ${ }^{115}$ che, proprio per la loro diffusione, entravano inevitabilmente nelle

Broncart en Souverain Pont, 1777]; Idem, Instit., ou Droit ecclesiast., 8, vol. 2, Paris, 1771 [C. Fleury, Institution au droit ecclesiastique, à Paris, chez Herissant fils, libraire, rue s. Jacques, a S. Paul \& a s. Hilaire, 1771]; Idem, Catechisme, 8, Lyon, 1786 [C. Fleury, Catechisme historique, contenant en abrege l'bistoire sainte, et la doctrine chretienne, à Lyon, chez Jean-Marie Bruyset Pere \& Fils, rue Saint-Dominique, 1786]; Idem, Discours sur l'Histoire ecclesiast., 8, Paris, 1763 [C. Fleury, Discours sur l'bistoire ecclesiastique, à Paris, chez Jean-Thomas Herissant, 1763]; Idem, id, 8, Porto, 1774 [C. Fleury, Discours sur l'histoire ecclesiastique, à Porto, chez F. Clamopin Durand, 1774].

110 Bossuet, Oeuvres, 8, vol. 22, Liege, 1766 [J.B. Bossuet, Oeuvres, à Liege, chez les Libraires Associes, 1766-1768]; Idem, Sermons, 8, vol. 9, Paris, 1772 [J.B. Bossuet Sermons, à Paris, chez Antoine Boudet, 1772]; Idem, Storia universal., 8, vol. 2, Ven., 1774 [J.B. Bossuet Discorso sopra la storia universale, Venezia, presso Carlo Todero, 1779]; Idem, Histoire des variations des Ec. protest., 4, vol. 2, Paris, 1688 [J.B. Bossuet Histoire des variations des eglises protestantes, à Paris, chez la veuve de Sebastien MabreCramoisy 1688]; Idem, Politique tirèe de l'Ecriture Sante, 12, vol. 2, Brux., 1721 [J.B. Bossuet, Politique tirée des propres paroles de l'Ecriture-Sainte, à Bruxelles, chez Jean Leonard, 1721]; Idem, Oraisons funébres, 8, Rouen, 1786 [J.B. Bossuet Recueil des oraisons, à Rouen, chez Jean Racine, 1786]; Idem, Orazioni funebri, 8, Venezia, 1748 [J.B. Bossuet Orazioni funebri, in Venezia, presso Giovanni Tagier, 1748]; Idem, Oraisons funébres, 12, Paris, 1741 [J.B. Bossuet Recueil des oraisons funebres pronucées à Paris, chez Jean De Saint, 1741]; Idem, Discours sur l'Histoire univers., 12, vol. 2, Paris, 1763 [J.B. Bossuet Discours sur l'Histoire universelle, à Paris, chez De Saint et Saillant, 1763]. 111 Racine, Storia ecclesiastica, 4, Vol. 21, Firenze, 1784 [B. Racine, Storia ecclesiastica divisa per secoli con riflessioni, Firenze, a spese di Francesco Pisoni, 1778. 1784]; Idem, Histoire eccles. avec les notes, 12, Vol. 14, Liege, 1751 [B. Racine, Abrégé de l'bistoire ecclesiastique, à Cologne, aux depens de la Compagnie, 1752-1762].

112 Berruyer, Histoire du peuple de Dieu avec notes, 8, vol. 10, Paris, 1742 [I.J. Berruyer, Histoire du peuple de Dieu, à Paris, chez Huart, 1742]; Idem, Storia de popolo di Dio, 4, vol. 6, Venezia, 1755 [I.J. Berruyer Storia del popolo di Dio, in Venezia, presso Giambattista Recurti, 1755].

113 Royaumont, Histoire du vieux, et du nouveau Testament, 8, Paris, 1755 [N. Fontaine, Histoire du Vieux et du Nouveau Testament avec des explications édifiantes... Par le sieur de Royaumont. Nouvelle édition, à Paris, chez P. Le Petit, 1755].

114 C. Fantappiè, Introduzione storica al diritto canonico, Bologna, Il Mulino, 1999, 190

115 Nelle biblioteche è significativa la presenza di testi illuministici, ad esempio nella raccolta di Alessando Verri. Cfr. F. Tarzia, La letteratura nobiliare a Roma nella 
case di quella classe di lettori in costante aumento per tutto il Settecento. Un nucleo fondamentale è costituito dal pensiero illuministico francese. Di Voltaire ${ }^{116}$ e Rousseau ${ }^{117}$ sono state acquistate le raccolte generali. Tra gli altri autori più apprezzati, si ricordano D'Aguessau, ${ }^{118}$ Condillac, ${ }^{119}$

seconda metà del Settecento: aspetti e proposte di metodo, in G. Tortorelli (a cura di), Biblioteche nobiliari e circolazione del libro tra Settecento e Ottocento, Bologna, Pendragon, 2002, 219-229.

116 Voltaire, Oeuvres collect. compl., 8, Vol. 57, Londra, 1781 [Collection complette des ceuvres de Mr. de Voltaire, [Lausanne, F. Grasset], 1770-1781]; Idem Vita di Carlo XII, 8, Vol. 2 in 1, Venezia, 1761 [Voltaire, Storia di Carlo XII re di Svezia, in Venezia, presso Francesco Pitteri, 1761]; Idem, Theatre, 12, Vol. 10, Londres, 1782 [Voltaire, Théâtre de Voltaire, à Londres, 1782]; Idem, Lettres, 8, Vol. 12, 1785 [Voltaire, Recueil des lettres de M. de Voltaire, De l'Imprimerie de la Société littéraire typographique, 1785]; Idem, Lettres du Roy de Prusse, 8, Vol. 3, 1785 [Voltaire, Recueil des lettres de M. de Voltaire et du roi de Prusse, De l'Imprimerie de la Société littéraire typographique, 1785]; Idem, Lettres de M. D'Alambert, 8, Vol. 2, 1785 [Voltaire, Recueil des lettres de M. de Voltaire et de M. d'Alembert, De l'Imprimerie de la Société littéraire typographique, 1785]; Idem, Lettres de l'Imperatrice de Russie, 8, 1785 [Voltaire, Recueil des lettres de M. de Voltaire et de l'impératrice de Russie, De l'Imprimerie de la Société littéraire typographique, 1785]; Idem, La Pucelle d'Orleans, 12, Geneve, 1777 [Voltaire, La pucelle d'Orleans, poeme heroi-comique, en dix-buit chants, à Geneve, 1777]; Idem, Poemes Epitres et autres Poesies, 12, Geneve, 1777 [Voltaire, Poèmes, épîtres et autres poésies par M. de Voltaire, à Genève, 1777].

117 Rosseau, Oeuvres, 12, Vol. 34, Geneve, 1789 [Rousseau, Collection complete des oeuvres de J.J. Rousseau, citoyen de Geneve, Geneve, 1782-1789]. Sulla ricezione del pensatore ginevrino in Italia: V. Ferrone, I profeti dell'Illuminismo, Bari, Laterza, 2000, 302-303.

118 Aguesseau, Lettres philosophiques, 12, vol. 3, Yverdon, 1785 [H.F. d'Aguesseau, Lettres philosophiques, fragmens et reflexions sur Jesus-Christ, Yverdon, 1785]; Idem, Meditations philosophiques, 12, vol. 4, Yverdon, 1780 [H.F. d'Aguesseau, Meditations philosophiques sur l'origine de la justice, Yverdon, 1780]; Idem, Oeuvres, 8, vol. 7, Yverdon, 1764 [H.F. D'Aguesseau, Oeuvres de M. le Chancelier d'Aguesseau, Yverdon, s.n., 1763-1764]; Idem, Discours, 12, vol. 2, Amsterdam, 1757 [H.F. d'Aguesseau, Discours et autres ouvrages, Amsterdam, 1757].

119 Condillac, Origine della umana cognizione, 8, vol. 2 in uno, Roma, 1784 [E. Bonnot de Condillac, Saggio dell' abate di Condillac accademico di Berlino sopra l'origine delle umane cognizioni, in Roma, nella stamperia di Giovanni Zempel, 1784]; Idem, Le Commerce, 8, vol. 2 in uno, Amsterd., 1776 [E. Bonnot de Condillac, Le commerce et le gouvernement, considere relativement l'un à l'autre, à Amsterdam, et se trouve à Paris, chez Jombert \& Cellot, libraires, rue Dauphine, 1776]; Idem, Cours d'Etude, 8 , vol. 12, Geneve, 1780 [E. Bonnot de Condillac,Cours d'étude pour l'instruction du Prince de Parme, Genève, chez Du Villard Fils \& Nouffer, 1780]; Idem, Oeuvres, 12, vol. 3, Paris, 1787 [E. Bonnot de Condillac, Oeuvres, à Paris, chez les Libraires associes, 1787]. 


\begin{abstract}
Montesquieu, ${ }^{120}$ Thomas, ${ }^{121}$ Raynal, ${ }^{122}$ La Bruyère ${ }^{123}$ e Mably. ${ }^{124}$ Completa questo elenco l'Encyclopedie di Diderot e D'Alembert. ${ }^{125}$ Venendo ora
\end{abstract}

120 Idem Persanes, 12, vol. 2 in 1, Cologne, 1739 [C.L. de Montesquieu, Lettres persanes, à Cologne, chez Pierre Marteau, 1739]; Montesquieu, Oeuvres, 8, vol. 6, Amsterdam, 1764 [C.L. de Montesquieu, Oeuvres, à Amsterdam et à Leipzig, chez Arkstee \& Merkus, 1764]; Idem, Lettres, 8, 1767 [C.L. de Montesquieu, Lettres familieres, 1767].

121 Thomas, Oeuvres diverses, 12, Vol. 2, Amsterd., 1772 [A.L. Thomas, Oeuvres diverses de M. Thomas, à Amsterdam, chez E. van Harrevelt, 1772]; Idem, Elogi, 8, Vol. 2, Parma, 1772 [A. L. Thomas, Elogi, in Parma, presso Filippo Carmignani, 1772]; Idem, Oeuvres, 12, Vol. 4, Amsterd., 1773 [A.L. Thomas, Oeuvres, Amsterdam e se trouve a Paris, chez Moutard, libraire de madame La Dauphine, rue du Hurepoix, a St. Ambroise, 1773]; Idem, Carattere delle donne colle note, 12, Venezia, 1773 [A.L. Thomas, Saggio sopra il carattere i costumi e lo spirito delle donne ne' varii secoli, Venezia, appresso Giovanni Vitto, 1773].

122 Raynal, Histoire de Isle Franc., 8, Lausanne, 1784 [G.T.F. Raynal, Histoire philosophique et politique des isles françoises dans les Indes occidentales, à Lausanne, chez J. Pierre Heubach \& Comp., 1784]; Idem, Storia philosophica e politica, 8, Vol. 18, 1777 [G.T.F. Raynal, Storia filosofica e politica degli stabilimenti, e del commercio degli Europei nelle due Indie. Opera dell' abate Raynal, 1776-1777]; Idem, Histoire philosof et polit., Vol. 7 in 4, Amsterd., 1772 [G.T.F. Raynal, Histoire philosophique et politique des etablissements et du commerce des europeens dans les deux Indes, Amsterdam, 1772]; Idem, Oeuvres, 8, Vol. 4, Geneve, 1784 [G.T.F. Raynal, Oeuvres de m. l'Abbe Raynal, à Geneve, chez J.L. Pellet, imprimeur de la Ville \& de l'Academie, 1784]; Idem, Tableau ou Revolution de Cologn. Angl., 12, Vol. 2 in 1, Amterdam, 1781 [G.T.F. Raynal, Tableau et revolutions des colonies angloises dans l'Amerique septentrionale, à Amsterdam, chez la Compagnie des libraires, 1781]. Su Raynal: K. Ohji, La fin de l'Ancien Régime en Europe selon l'"Histoire de deux Indes", in A. Lilti - C. Spector (éds.) Penser l'Europe au XVIIIe siècle. Commerce, Civilisation, Empire, Oxford, Voltaire Foundation, 2014, 117-136.

123 De la Bruyere, Carattere di Teofrasto colle note, 8, vol. 6, Venezia, 1759 [J. de La Bruyere, I caratteri di Teofrasto, coi caratteri, o costumi di questo secolo, in Venezia, appresso Giambattista Novelli, 1758-1759]; Idem, Les Caracteres, 12, vol. 2, Paris, 1750 [J. de La Bruyere, Les caracteres de Mr. de La Bruyere, à Paris, chez David pere libraire Quay des Augustins a la Providence, 1750]; Idem, Maximes et Reflexions, 12, Geneve, 1782 [J. de La Bruyere, Maximes et reflexions morales extraites de La Bruyere, à Geneve, 1782]; Theophraste, Les Caracteres, 8, Paris, 1696 [J. de La Bruyere, Les caractères de Théophraste, à Paris, chez Estienne Michallet, 1696].

124 Mably, Oeuvres, 8, vol. 10, Haye, 1767; Idem, Des Droits, et des Devoirs du Citoyen, 12, Paris, 1789 [G. de Mably, Des Droits et des devoirs du citoyen, ParisLausanne, chez F. La Combe, 1789].

125 Encyclopedie par M. Diderot et d'Alambert, 8, vol. 36, Lus., 1781 [Encyclopedie, ou Dictionnaire raisonne des sciences, des arts et des metiers, à Lausanne et a Berne, chez les Societes typographiques]; Idem Recueil des Planches, 4, vol. 3, 
all'Italia, letture di illuministi italiani sono gli Elementi di morale di Gian Rinaldo Carli, ${ }^{126}$ le Ricerche sulla scienza dei governi di Giuseppe Gorani, ${ }^{127}$ le opere di Antonio Genovesi ${ }^{128}$ e La scienza della legislazione di Gaetano Filangieri. ${ }^{129}$ Uno sguardo va poi gettato all'illuminismo lombardo, alla

Lusane, 1781. Tra le opere di divulgazione vanno inclusi i dizionari (R. Birn, Enciclopedismo, in V. Ferrone - D. Roche (a cura di), L'Illuminismo. Dizionario storico, Bari, Laterza, 1997, 180): «Per tutto il secolo una moltitudine di dizionari, lessici e teatri meccanici aveva precorso l'opera di Diderot, ognuno di essi evidenziando delle caratteristiche che egli avrebbe poi assimilato e modificato». Si contano ben 20 dizionari, di cui la metà in lingua francese, oltre a: Hofmanni, Lexicon univol., fol., vol. 4, Lugd. Batav., 1698 [J.J. Hofmann, Lexicon universale historiam sacram et profanam omnis aevi, omniumque gentium, Lugduni Batavorum, apud Jacob. Hackium, Cornel. Boutesteyn, Peter Vander AA, \& Jord. Luchtmans, 1698].

126 Elementi di morale, 12, Pisa, 1756 [G.R. Carli, Elementi di morale, in Pisa, nella nuova stamperia di Gio. Paolo Giovannelli, e compagni, si vendono in Firenze dal Bonajuti libraio, 1756].

127 Scienza dei Governi, 8, Vol. 2, Lusanna, 1790 [G. Gorani, Ricerche sulla scienza dei governi, Lausanna, appresso Heubach, Durand e Comp., 1790].

128 Genovesi, Lettere famigliari, 8, vol. 2 in 1, Venezia, 1775 [A. Genovesi, Lettere familiari, in Venezia, presso Pietro Savioni, 1775]; Idem, Lezioni di Commercio, 8, Bassano, 1769 [A. Genovesi, Lezioni di commercio o sia d'economia civile, Bassano, a spese Remondini di Venezia, 1769]; Idem, Diceosina, 8, vol. 3, Napoli [A. Genovesi, Della diceosina o sia della filosofia del giusto e dell'onesto, Napoli, Domenico Terres negoziante di libri]; Idem, Elementi dell'arte logico-crit., 12, Ven., 1776 [A. Genovesi, Gli elementi dell' arte logico-critica, in Venezia, presso Domenico Pompeati, 1776]; Idem, Meditazioni filosofiche sulla Relig., 8, Napoli, 1766 [A. Genovesi, Meditazioni filosofiche sulla religione, e sulla morale, Napoli, a spese Remondini, 1766]; Idem, Elementa theolog., 4, vol. 2, Venet., 1771 [A. Genovesi, Universae christianae theologiae elementa dogmatica, bistorica, critica, Venetiis, typis Jo. Baptistae Pasquali, 1771]; Idem., Logica, Bassano, 1766 [A. Genovesi, La logica per gli giovanetti, nella stamperia di Bassano, a spese Remondini, 1766]. Il pensiero di Genovesi ebbe molto influenza in tutta la penisola: V. Ferrone, La società giusta ed equa. Repubblicanesimo e diritti dell'uomo in Gaetano Filangieri, Bari, Laterza, 2008, 100-123; A. Trampus, Storia del costituzionalismo italiano nell' età dei lumi, Bari, Laterza, 2009, 149-155.

129 Filangieri, Scienza della Legislazione, 8, vol. 8 in 4, Napoli, 1780 [G. Filangieri, La scienza della legislazione del cavalier Gaetano Filangieri, Napoli, nella stamperia Raimondiana, 1780-1789]. Sulla Scienza della legislazione: R. Ajello, L'estasi della ragione. Dall'illuminismo all'idealismo. Introduzione alla Scienza di Filangieri, in Gaetano Filangieri e l'illuminismo europeo, Napoli, Guida, 1991, 13-145; su Filangieri: A. Trampus (a cura di), Diritti e costituzione. L'opera di Gaetano Filangieri e la sua fortuna europea, Il Mulino, Bologna 2005; sull'importanza dell'opera nel contesto del tardo illuminismo, V. Ferrone, Storia dei diritti dell'uomo, Bari, Laterza, 2015, 341-348. 
Milano del «Caffè». ${ }^{130}$ I protagonisti indiscussi di quegli anni sono i fratelli Verri ${ }^{131}$ e Beccaria. ${ }^{132}$ Mentre sorprende che non vi sia nessuna traccia dei primi nel catalogo, due sono le copie de' Dei delitti e delle pene, ${ }^{133}$ edizioni che comprendono il commento di Voltaire e le note del Facchinei. ${ }^{134}$

Per inciso, l'opera del Beccaria non è l'unico libro proibito in possesso dei Visconti, ben al corrente di quali siano gli autori colpiti dalla censura ecclesiastica ${ }^{135}$ ed austriaca: ${ }^{136}$ poco sopra si sono menzionati

130 F. Venturi, Settecento Riformatore, I, Da Muratori a Beccaria. 1730-1764, Torino, Einaudi, 1969, 645-747; D. Carpanetto - G. Ricuperati, L'Italia del Settecento, Bari, Laterza, 1986, 323-340.

131 Su Pietro Verri: C. Capra (a cura di) Pietro Verri e il suo tempo, a cura di Carlo Capra, Bologna, Cisalpino, 1999; C. Capra, I progressi della ragione: vita di Pietro Verri, Bologna, Il Mulino, 2002.

132 Su Beccaria, senza pretesa di completezza: M.A. Cattaneo, Cesare Beccaria e l'illuminismo giuridico europeo, in Cesare Beccaria tra Milano e l'Europa, cit., 196-224; F. Venturi, Settecento riformatore. I. Da Muratori a Beccaria, cit., I, 707-715; M. Pisani, Cesare Beccaria e l'Index librorum probibitorum, Napoli, ESI, 2013.

133 Beccaria, Dei delitti e delle pene coi commenti di Voltaire, 8, vol. 2, Venezia, 1781 [C. Beccaria, Dei delitti e delle pene, in Venezia, appresso Rinaldo Benvenuti, 1781]; Idem, Dei delitti e delle pene colle note, vol. 2, 8, Bassano, 1789 [C. Beccaria, Dei delitti e delle pene edizione novissima in quattro tomi ridotta, Bassano, a spese Remondini di Venezia, 1789]. Un nuovo bilancio storiografico sull'opera è tracciato in: G. Rossi - F. Zanuso (a cura di), Attualità e storicità del «Dei delitti e delle pene» a 250 anni dalla pubblicazione, Napoli, ESI, 2015.

134 Il tema della tortura deve aver incuriosito i Visconti, che hanno letto anche il libro di Sonnenfels, Della Tortura, 8, Milano, 1776 [J. von Sonnenfels, Su l'abolizione della tortura del sig. Di Sonnenfels consigliere ... Tradotto dal tedesco. Con alcune osservazioni sul medesimo argomento, Milano, appresso Giuseppe Galeazzi R. stampatore, 1776]. Il dibattito sulla riforma del processo penale ha in Beccaria il suo promotore, e impegna la generazione di giuristi che opera a partire dal seconda metà del XVIII secolo. Oltre al citato Cremani, i Visconti possiedono la celebre opera De ordine seu forma judiciorum criminalium diatriba di Filippo Maria Renazzi in prima edizione [F. Renazzi, De ordine seu forma judiciorum criminalium diatriba ..., Romae, Joannes Generosus Salomoni, 1777]. Cfr. E. Dezza, Note su accusa e inquisizione nella dottrina settecentesca, ora in Id., Saggi di storia del diritto penale moderno, Milano, Led, 1992, 22-29.

135 Due, infatti, le edizioni dell'Index librorum prohibitorum [Index librorum probibitorum sanctissimi D.N. Benedicti XIV, Romae, ex typographia Revol. Camerae Apostolicae, 1758; Index librorum probibitorum sanctissimi d.n. Benedicti XIV, Parmae, apud Philippum Carmignani, typographum ex privilegio s.r.c., 1783]. Sulla censura ecclesiastica: E. Rebellato, La fabbrica dei divieti. Gli indici proibiti da Clemente VIII a Benedetto XIV, Milano 2008; P. Delpiano, Il governo della lettura. Chiesa e libri nell'Italia del Settecento, Bologna, Il Mulino, 2007; Ead., Il controllo ecclesiastico della lettura nell'Italia dei Lumi, in E. Tortarolo (a cura di), La censura nel secolo dei Lumi. Una visione internazionale, Torino, UTET, 2011, 65-93.

136 A. Tarchetti, Censura e censori di sua maestà imperiale nella Lombardia 
Sarpi, Montesquieu, ma la lista si allunga con Machiavelli, ${ }^{137}$ Locke $^{138} \mathrm{e}$ Bayle. ${ }^{139}$ Questi rilievi consentono di includere i Visconti in quella «zona franca» rappresentata da un' «aristocrazia di lettori» ${ }^{10}$ con libero accesso alle opere condannate. Per Pavia occorre menzionare, ad esempio, il marchese Giuseppe Gaspare Belcredi, che, forse per il suo ruolo di censore, possedeva e consultava regolarmente libri caduti sotto la scure della censura. ${ }^{141}$ Del resto, per descrivere la situazione dopo l'e-

austriaca (1740-1780), in Economia, istituzioni, cultura, cit., II, 741-792; A.P. Montanari, Il controllo della stampa, «ramo di civile polizia». L'affermazione della censura di stato nella Lombardia Austriaca del XVIII secolo, Roma moderna e contemporanea, 2 (1992), 343 378. La creazione di apparati di censura di Stato a discapito di quelli ecclesiastici di matrice post tridentina è analizzata, attraverso le diverse realtà italiane, da: L. Braida, Censure et circulation du livre en Italie au XVIII e siècle, JMEH 3 (2005), 81-98; specificamente, per la Toscana: S. Landi, Il governo delle opinioni. Censura e formazione del consenso nella Toscana del Settecento, Bologna, Il Mulino, 2000; Id., I regimi della censura nella Toscana del Settecento, in La censura nel secolo dei Lumi, 95-113; per il Regno di Napoli: M.C. Napoli, Letture proibite. La censura dei libri nel regno di Napoli, Milano, Franco Angeli, 2002; G. Imbruglia, Censura e giurisdizionalismo nel secondo Settecento a Napoli. Il Delegato alla Reale Giurisdizione, in La censura nel secolo dei Lumi, cit., 115-147.

137 Machiavelli, Opere, 4, vol. 6, Firenze, 1783 [N. Machiavelli, Opere di Niccolo Machiavelli, in Firenze, a spese di Gaetano Cambiagi libraio, 1782-1783]; Idem, Opere inedite, 4, vol. 3, Londra, 1777 [N. Machiavelli, Opere inedite in prosa, e in verso di Niccolo Macchiavelli, Londra, 1777]; Idem, Le Prince avec un comment., 12, Amsterd., 1683 [N. Machiavelli, Le Prince de Nicolas Machiavel, à Amsterdam, chez Henry Wetstein, 1683].

138 Idem, Le christianisme raisonnable, 12, vol. 2, Amsterd., 1740 [J. Locke- P. Coste, Le christianisme raisonnable, tel qu'il nous est represente dans l'Ecriture Sainte, à Amsterdam, chez Zacharie Chatelain, 1740]. Di Locke sono pure presenti: Locke, Gouvernement civil., 8, Amsterdam, 1755 [J. Locke, Du gouvernement civil, à Amsterdam, chez J. Schreuder et Pierre Mortier le Jeune, 1755]; Idem, L'Entendement humain, 8, vol. 4, Amsterdam, 1774 [J. Locke, Essai philosophique concernant l'entendement humain, à Amsterdam, aux depens de la Compagnie, 1774].

139 Idem, Oeuvres, fol., vol. 4, Haye, 1731 [P. Bayle, Oewvres diverses de Mr.

Pierre Bayle, à La Haye, chez P. Husson, T. Johnson, P. Gosse, 1727-1731]; Response aux question d'un Provincial, 12, Vol. 5, Rotterd., 1707 [P. Bayle, Reponse aux Questions d'un Provincial, à Rotterdam, chez Reiniers Leers, 1704-1707].

140 A. Rotondò, La censura ecclesiastica e la cultura, in Storia d'Italia, V.1, Torino, Einaudi, 1973, 1415.

141 Pare qui curioso segnalare che nel fondo Visconti si trova un libro con applicata sul frontespizio una striscia di carte a stampa, la quale indica la provenienza del volume proprio dalla biblioteca di Giuseppe Gaspare Belcredi: G. Courtilz de Sandras, La vie de Jean-Baptiste Colbert ministre d'etat sous Louis XIV roy de France, à Cologne chez Pierre Le Vray, 1696. 
manazione dell'editto del 30 dicembre 1768, con cui l'imperatrice toglieva alla Chiesa ed al Senato il controllo sulla legittimità delle stampe, resta ancora valido il bilancio di Pietro Verri, tracciato pochi mesi prima della riforma, ove si loda l'estrema «libertà per i libri che si godeva in Lombardia». ${ }^{142}$

Volendo riannodare i fili di questa panoramica sulla biblioteca Visconti, si osserva come i titoli del catalogo consentano di apprezzare l'apertura culturale di questa famiglia. La biblioteca rispecchia, infatti, la vasta proiezione di interessi dei Visconti, che spaziano dal diritto alle scienze storiche e umane. Essi appaiono, in definitiva, tutt'altro che operatori del diritto "monodimensionali", intenti alla pratica notarile e forense.

Allo stesso tempo, la biblioteca restituisce la dimensione culturale cittadina nella quale questa famiglia si colloca. Pavia dimostra di ricevere, nel corso del Settecento, linfa vitale dal processo di rinnovamento dell'Università, rifondata da Maria Teresa. La città non è estranea a quanto accade in Italia ed più in generale in Europa, e i numerosi volumi dei philosophes sono l'attestazione di quanto essa riesca ad inserirsi nel più ampio scenario storico generale.

4. Queste conclusioni aiutano a chiarire un aspetto non secondario della personalità di Emilio Gabba, studioso sia di storia locale ${ }^{143}$ che della storiografia europea sul mondo antico. L'attenzione e la curiosità intellettuale che egli dedicò, in parallelo, alla sua città ed agli storici e ai pensatori del Settecento mi pare possano avere avuto, almeno embrionalmente, inizio nella quotidiana frequentazione della biblioteca Visconti nella casa di famiglia.

Di questa consuetudine ne dà ulteriore testimonianza una recente scoperta ad opera del fratello di Emilio Gabba, l'ingegnere Alberto. Si tratta del Regolamento della biblioteca che Gabba, appena ventenne, aveva redatto nel settembre del 1947. Esso consta di 10 arti-

142 Lettera Pietro Verri a Alessandro Verri 9.4.1768, citata in A.P. Montanari, Vendere e comprare libri nella Milano del Settecento (1700-1789), Storia in Lombardia, 20 (2000), 35.

143 D. Mantovani, Emilio Gabba e la storia locale: fra storia generale e civismo, in Emilio Gabba: un maestro della storia antica cit., 137-156. 
coli e si affianca ad una planimetria della biblioteca. La stesura del regolamento coincide, inoltre, con la lunga opera di catalogazione del fondo librario. Gabba, infatti, ritagliando fogli già usati, come era sua abitudine fare, aveva creato quasi due migliaia di schede bibliografiche, raccolte in piccole cartelle di color rosso, che aveva poi ordinato, per autore, alfabeticamente.

Il regolamento, redatto dal Bibliotecario Direttore - è infatti così che Gabba si qualifica - è ancora in vigore; e mi pare significativo qui ricordare le norme a cui gli utenti devono attenersi:

1. La biblioteca non ammette il prestito domiciliare, salvo in casi straordinari, dietro autorizzazione dei proprietari e del bibliotecario direttore.

2. L'uso dei libri è ammesso in sede sotto la sorveglianza del Bibliotecario direttore.

3. Il pubblico può accedere al catalogo, ma non alla scaffalatura.

4. É severamente vietato correggere il catalogo o scrivere, manomettendoli, sui libri. Eventuali errori sul catalogo saranno segnalati al bibliotecario direttore, che provvederà in conseguenza.

5. Chi si renderà colpevole di furto o manomissione sarà escluso per sempre dall'uso della biblioteca.

6(8). La lettura degli incunaboli elencati a parte è proibita se non sotto sorveglianza.

7(9). Il bibliotecario direttore è autorizzato a negare $i$ libri, che ritenga non adatti o troppo preziosi.

8(10). I manoscritti non si danno in lettura che dietro speciale autorizzazione dei proprietari.

9(11). In biblioteca non si può fumare. Non vi si può accedere con candele o mezzi di illuminazione a fiamma.

10(12). L'orario è il seguente: Mattina 9-12; Pomeriggio 15-17. Nei giorni festivi è chiusa. Annualmente dal 1 al 31 agosto si chiude per la pulitura.

Rileggendo nuovamente il regolamento, ho potuto verificare quante trasgressioni il clemente Bibliotecario Direttore mi consentì durante lo studio della biblioteca, ennesimo privilegio che volle riservarmi e per il quale rinnovo qui grata ed affettuosa riconoscenza. 


\section{RINGRAZIAMENTI}

Desidero esprimere il mio ringraziamento al Presidente dell'Istituto Lombardo, professor Beretta, al professor Mantovani e agli altri membri del Comitato scientifico, nelle persone dei professori Laffi, Mazzoli, Troiani, Vitale, per l'invito a partecipare all'incontro di studio in ricordo di Emilio Gabba. Sono inoltre grato all'ingegnere Alberto Gabba per avermi consentito la consultazione di documenti e materiali familiari.

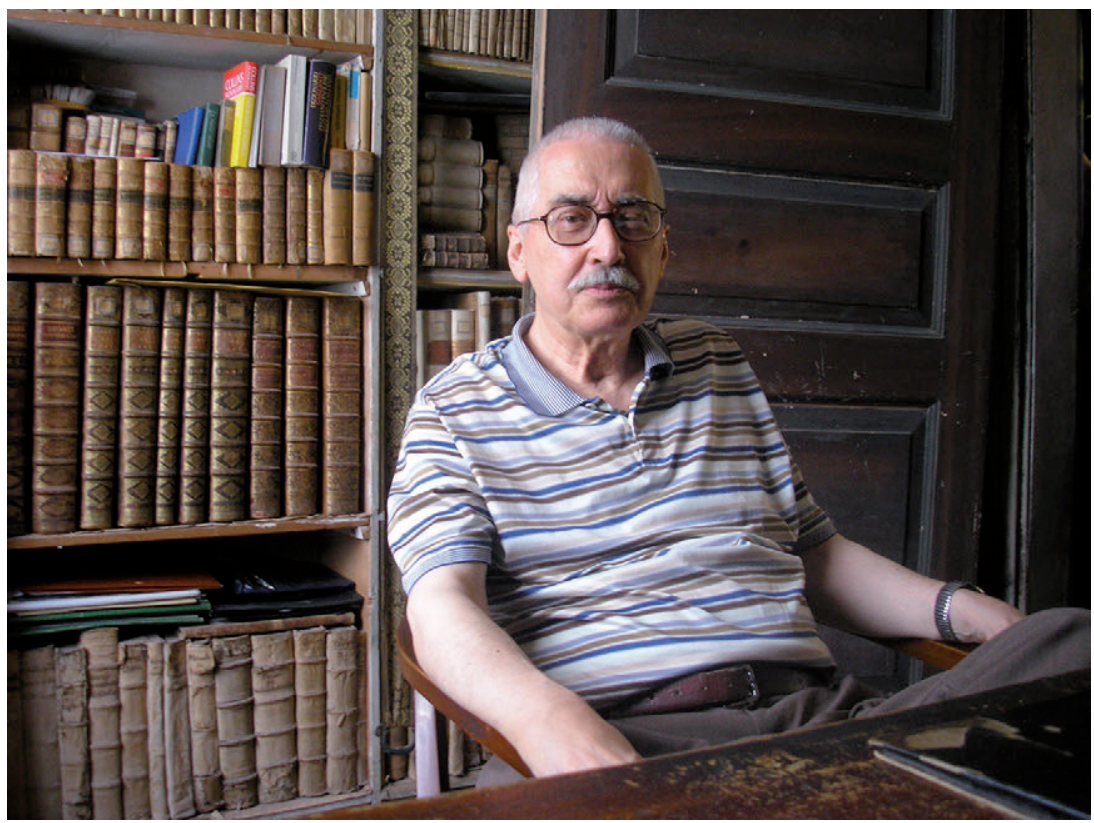

Emilio Gabba, seduto alla scrivania nella sua biblioteca. 


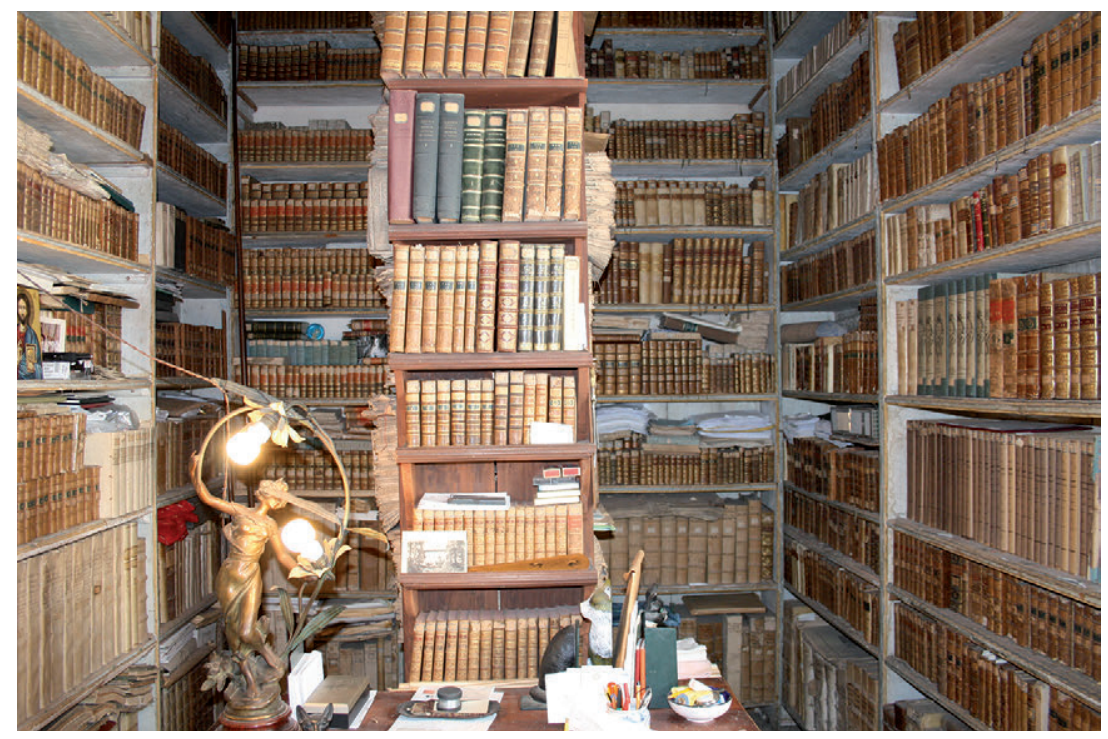

Prospettiva della collezione libraria dei notai Visconti in casa Gabba.

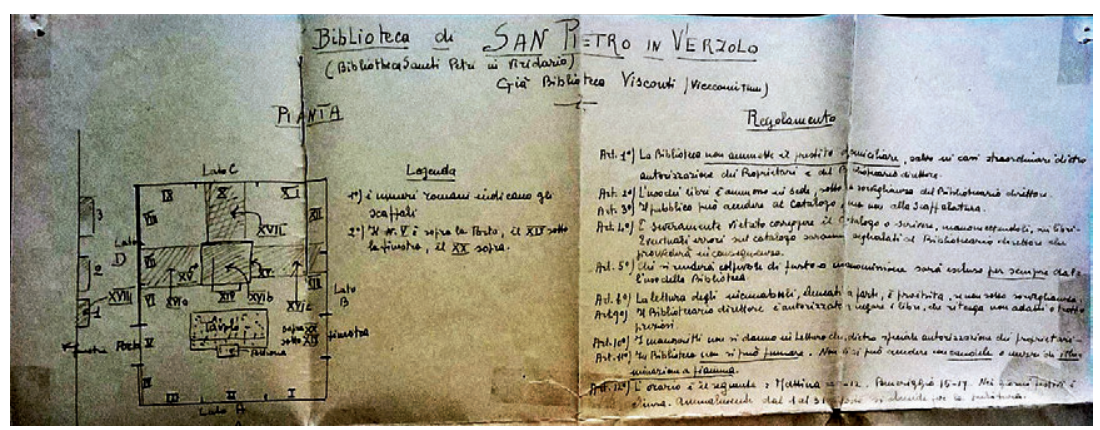

Regolamento e planimetria della biblioteca Gabba. 


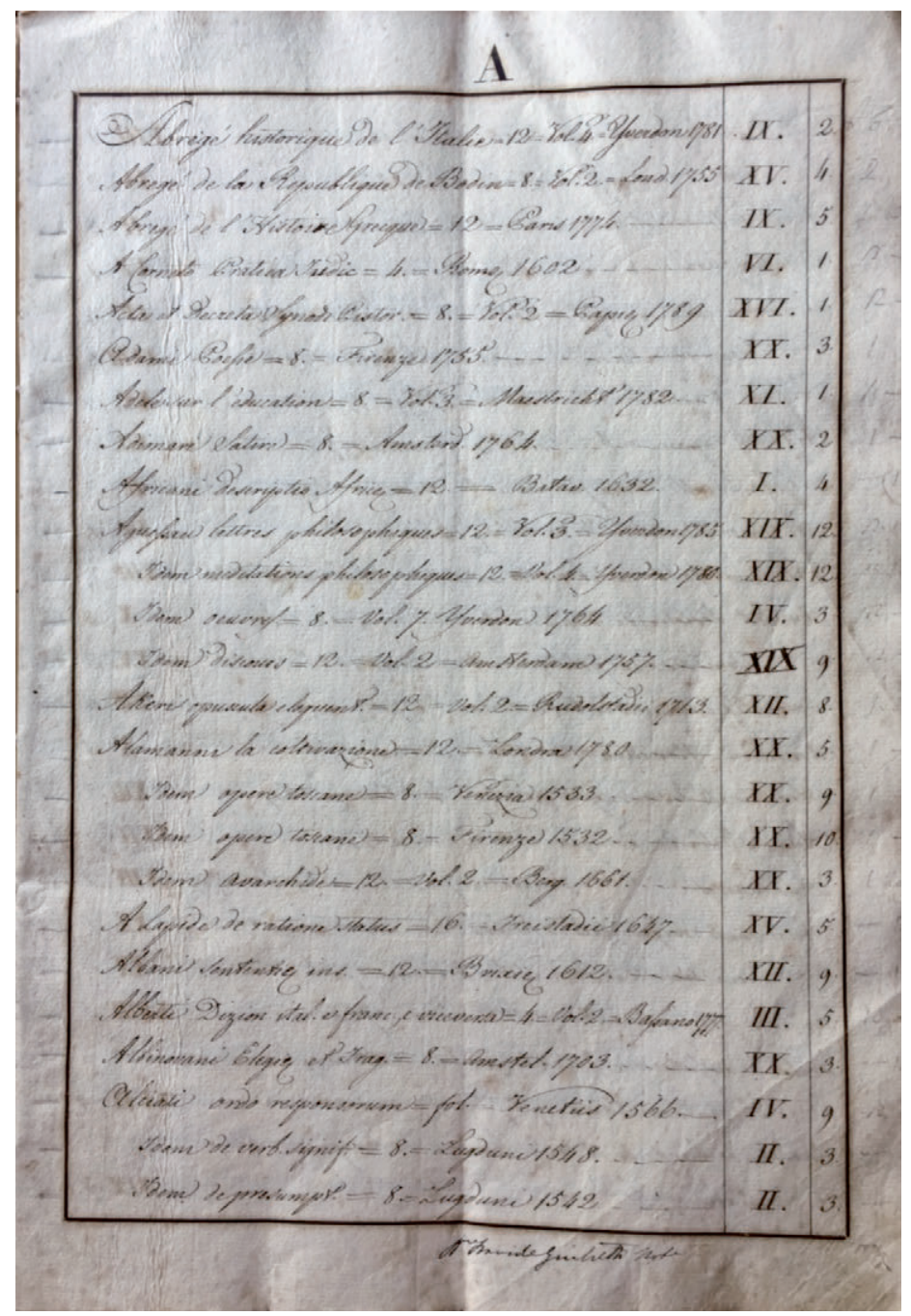

La prima pagina del catalogo librario settecentesco. 